\title{
Estimation of the Sensible and Latent Heat Fluxes by Reciprocal Analysis at an Arid and Semi-Arid Region
}

\author{
Toshisuke Maruyama*, Manabu Segawa \\ Faculty of Environmental Science, Ishikawa Prefectural University, Ishikawa, Japan \\ Email:^maruyama@ishikawa-pu.ac.jp,manabu@ishikawa-pu.ac.jp
}

How to cite this paper: Maruyama, T. and Segawa, M. (2017) Estimation of the Sensible and Latent Heat Fluxes by Reciprocal Analysis at an Arid and Semi-Arid Region. Open Journal of Modern Hydrology, 7, 38-64.

http://dx.doi.org/10.4236/ojmh.2017.71003

Received: October 12, 2016

Accepted: January 23, 2017

Published: January 26, 2017

Copyright $\odot 2017$ by authors and Scientific Research Publishing Inc. This work is licensed under the Creative Commons Attribution International License (CC BY 4.0).

http://creativecommons.org/licenses/by/4.0/ (c) (i) Open Access

\begin{abstract}
Sensible and latent heat flux at semi-arid and arid region, i.e., evapotranspiration, has been researched for long time because it serves an important role for water resource issues. However, the issues have not solved completely yet. Accordingly, by applying the Bowen ratio concept on the soil surface, the sensible and latent heat fluxes are reciprocally estimated using single height temperature $(T z)$ and humidity (rehz) with the net radiation $(R n)$ and heat flux into the ground $(G)$. The procedure proposed by authors initially estimates the soil surface temperature $(T s)$ and the relative humidity (rehs) using optimization techniques. The method is remarkably effective to expand for estimating evapotranspiration at various regions. The validity of the method is confirmed by the latent heat flux $(I E)$ and sensible heat flux $(H)$ observed by the eddy covariance method. The hourly change of the $I E, H, T s$ and rehs on the soil surface, yearly change of $I E$ and $H$ and relationship of estimated $I E$ and $H$ versus observed are clarified. Yearly change of evapotranspiration is also estimated. The analysis is performed by general method (1), conventional method and general method (2). Above results are very useful for water resources issue and irrigation planning. The research is conducted using hourly data at eight globally dispersed sites using FLUXNET.
\end{abstract}

\section{Keywords}

Bowen Ratio, Eddy Covariance, Evapotranspiration, Optimization,

Single Height Temperature and Humidity

\section{Introduction}

Recently, we firstly reported reciprocal analysis of sensible and latent heat fluxes in a forest and humid region [1] [2]. By applying the procedure, this research highlighted the same problems in "an arid and semi-arid regions". As is well known, the region usually faced to very severe water shortage. Therefore, reasonable estimation of latent 
heat flux (evapotranspiration) is very important issues. But the analysis in the local area still has a big problem because the flux severely affects local climate conditions.

Our proposed method is reciprocal analysis of the sensible and latent heat fluxes using single height temperature $(T z)$ and humidity (rehz) with net radiation $(R n)$ and heat flux into the ground $(G)$. This method can't be found out in the previous research except ours [1] [2]. This method is remarkably effective to extend the utilization for various regional areas.

One of the problems in the method is how to determine the initial values and constraints. This paper describes mainly focusing on this problems including estimation of those fluxes in arid and semi-arid region.

The results compare general solution (1) (solved two simultaneous equations that mathematically unified soil surface temperature $(T s)$ and humidity (rehs)), general solution (2) (solved one equation using $T s$ observed by radiometer that unified rehs mathematically) and conventional solution (solved one equation that not unified $T s$ and rehs mathematically). Furthermore, this research describes relationship between annual evapotranspiration and precipitation from the aspect of water balance.

Actually, the unknown variables in the proposed method, Ts and rehs, were estimated by the non-linear optimization technique known as the General Reduced Gradient (GRG) using the Excel Solver (Appendix 1) [1] [2].

\section{Methods}

\subsection{Method of Analysis}

\subsubsection{Fundamental Concept of the Model}

The fundamental concept of the method is quiet similar with previous research. Here, we describe the outline briefly. The proposed model considers the near-soil surface as shown in Figure 1. Net radiation moves from air to the soil surface, and it is portioned into sensible, latent and underground heat fluxes. $T s$ is the soil surface temperature, $T z$ is the air temperature at height $z, q(T z)$ is the specific moisture at height $z$, rehz is relative humidity in air at height $Z, q(T s)$ is the unsaturated specific moisture on the soil

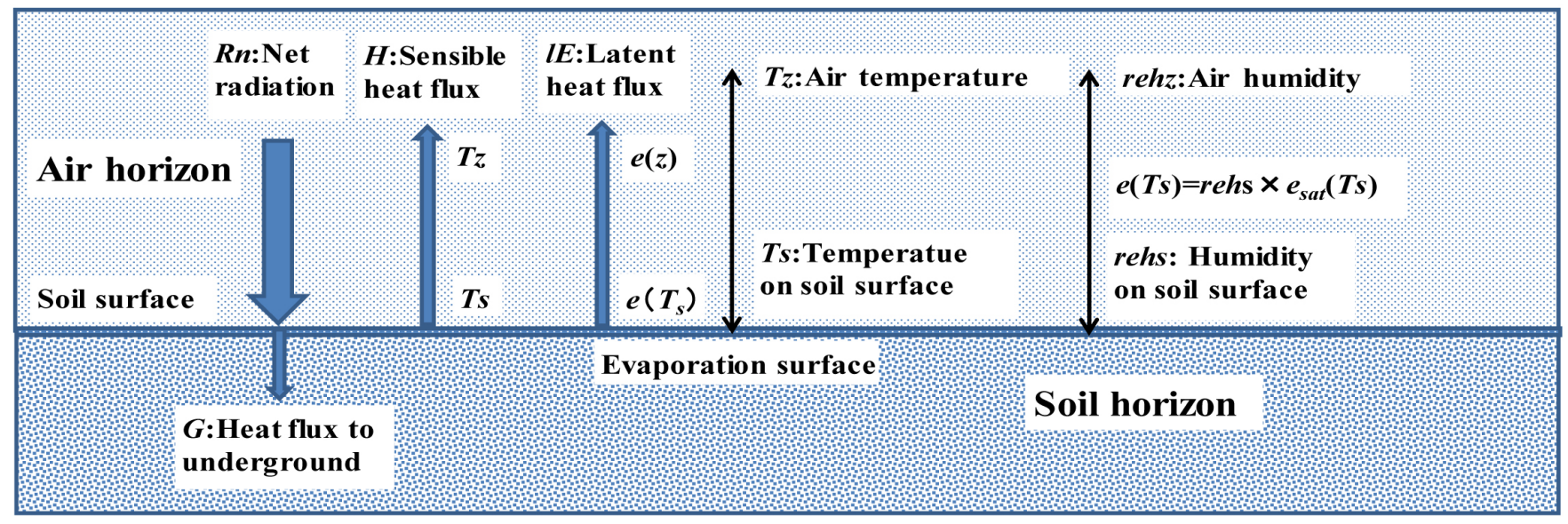

Figure 1. Schematic presentation of considered domain and used symbols [2]. (Symbols used in research defined as this figure. The net radiation $R n$ partitioned into ground $G$, sensible heat $H$ and latent heat $I E$. Thus, Heat balance relationship $R n=H+I E+G$ is always guaranteed). 
surface, and $q_{s a t}(T s)$ is the saturated specific moisture on the soil surface [1] [2].

The fundamental formulae of the model satisfy the following well-known heat balance relationship [3].

Heat balance relationship:

$$
R n=H+l E+G
$$

Here, $R n$ is the net radiation flux $\left(\mathrm{W} \cdot \mathrm{m}^{-2}\right), G$ is the heat flux into the ground $\left(\mathrm{W} \cdot \mathrm{m}^{-2}\right)$, $H$ is the sensible heat flux $\left(\mathrm{W} \cdot \mathrm{m}^{-2}\right)$, and $I E$ is the latent heat flux $\left(\mathrm{W} \cdot \mathrm{m}^{-2}\right)$.

In addition, the Bowen ratio $(H / I E)$ is defined as follows [1] [2] [3]:

$$
B_{0}=\frac{C p\left(T_{1}-T_{2}\right)}{l\left(q_{1}-q_{2}\right)}
$$

We apply the concept to the layer between the soil surface and observation height of $T z$ and rehz. However, the $T s$ and $q(T s)$ just on the surface are usually unknown.

\subsubsection{Equation for Determining the Unknown Variables Ts and rehs}

The purpose of the optimization is to determine the unknown variables $T s$ and $q(T s)$ without measurements. Initially, $T s$ and $q(T s)$, i.e., rehs $\times q_{s a t}(T s)$, are assumed. Then it's gradually improved according to the following optimization procedure [1] [2]:

$$
\begin{gathered}
R_{n}-G-H_{e s t, i}-l E_{e s t, i}=\varepsilon_{i}, i=0,1,2, \cdots, n \\
B_{a p p, i}=\frac{H_{e s t, i}}{l E_{e s t, i}}=\frac{C p\left(T_{s, a s s}-T_{z}\right)}{l\left[q\left(T_{s, a s s}\right)-q\left(T_{z}\right)\right]} \\
l E_{e s t, i+1}=\frac{R n-G}{1+B_{a p p, i}} \text { and } H_{e s t, i+1}=B_{a p p, i} \times l E_{e s t, i+1}
\end{gathered}
$$

Here, $i$ is number of iterations. $H_{\text {est }, i}$ is estimated sensible heat flux in $i$ times iteration, $l E_{e s t, i}$ is estimated latent heat flux of $i$ times iteration, $\varepsilon_{i}$ is residual of heat balance relationship of $i$ times iteration, $T_{\text {sass }}$ is assumed soil surface temperature, $q\left(T_{\text {sass }}\right)$ is assumed specific moisture at $T_{s a s s}, B_{a p p}$ is apparent ratio of sensible and latent heat flux under convergence process

Repeating the above calculation, the $B_{a p p}$ goes to $B_{0}$ according to the objective function where $\mathrm{ABS}\left(\varepsilon_{i}\right)$ reaches a minimum. Symbols used here presented in Figure 1.

Using the determined Bowen ratio $\left(B_{0}\right)$ after optimization, $I E$ and $H$ can be obtained as follows:

$$
l E_{e s t}=\frac{R n-G}{1+B_{0}} \text { and } H_{e s t}=B_{0} \times l E_{e s t} .
$$

Symbols used here presented in Figure 1.

To estimate $T s$, an adjustment factor $R T s$ was introduced using $T_{0}$ as follows:

$$
T_{s}=G \times D_{T_{o}} / K t \times R T s+T_{0}
$$

Here, $T_{0}$ is the observed soil temperature at a depth of $x \mathrm{~cm}, D_{T o}$ is the depth of the soil temperature observation, $K t$ is the assumed temperature conductivity $\left(\mathrm{W} \cdot \mathrm{cm}^{-1} \cdot{ }^{\circ} \mathrm{C}\right.$ ), Equation (7) describes how to estimate $T s$ by extrapolating $T_{0}$ using $G$, 
$D_{T o}$ and $K t[1][2]$.

\subsubsection{Correction of the Heat Imbalance by Multiple Regression Analysis}

The observed data have a heat imbalance that is well known as a "closure issue". Twine, et al. [4] indicated an approximately $10 \%-30 \%$ imbalance in $H$ and $I E$. Wilson, et al. [5] also indicated that approximately $20 \%$ of the imbalance is due to heat flux. Allen [6] suggested that the under-measurement of $I E$ is sometimes approximately $40 \%$.

Table 1 describes the imbalance in the heat energy. Therefore, the data should be correct. According to Allen's procedure, we corrected the data by multiple regression analysis using the observed data as follows [1] [2]:

$$
R n-G=A \times l E+B \times H .
$$

Here, $R n$ is the net radiation, $G$ is the heat flux into the ground, $I E$ is the latent heat flux observed, and $H$ is the sensible heat flux observed. $A$ is the regression coefficient for $I E$, and $B$ is the regression coefficient for $H$. The corrected data are expressed as $H_{c o r}$ for $H$ and $I E_{c o r}$ for $I E$. The observed data are expressed as $H_{o b s}$ and $I E_{o b s}$, and the imbalance data are also defined as $H_{i m b}\left(=R n-G-l E_{o b s}\right)$ for $H$ and $I E_{\text {imb }}\left(=R n-G-H_{\text {obs }}\right)$ for $I E$.

\subsubsection{Method of General Solution (1)}

To uniquely estimate the unknown variables $T s$ and $r e h s$, pair of two equations is required mathematically. We set the two equations as follows [1] [2]:

$$
\begin{gathered}
R_{n}^{j}-G^{j}-H_{e s t, i}^{j}-l E_{e s t, i}^{j}=\varepsilon_{i}^{j} \\
R_{n}^{j+1}-G^{j+1}-H_{e s t, i}^{j+1}-l E_{e s t, i}^{j+1}=\varepsilon_{i}^{j+1} \\
\varepsilon=\operatorname{ABS}\left[\varepsilon_{i}^{j}\right]+\operatorname{ABS}\left[\varepsilon_{i}^{j+1}\right] \text { goes to minimum. }
\end{gathered}
$$

Here, $j$ is the order of hours from 1 to the end of the analyzed hours and $i$ is the number of iterations.

The calculation is performed by solving Equation (9) and Equation (10) simultaneously under $T_{s}^{j}=T_{s}^{j+1}$ and $r e h s^{j}=r e h s^{j+1}$ conditions.

In addition, to prevent abnormal fluctuation of $H_{\text {est }}$ and $I E_{\text {est }}$ in the optimization process, constraints $R n-G<H, I E$ are applied. The reason described in previous research [1] [2]. The constraints for Equation (9) and Equation (10) are follows:

$$
\left.\begin{array}{l}
\left\{\operatorname{ABS}\left(H^{j}\right)+\operatorname{ABS}\left(H^{j+1}\right)\right\}<\left\{\operatorname{ABS}\left(R n^{j}-G^{j}\right)+\operatorname{ABS}\left(R n^{j+1}-G^{j+1}\right)\right\} \text { for } H \\
\left\{\operatorname{ABS}\left(l E^{j}\right)+\operatorname{ABS}\left(l E^{j+1}\right)\right\}<\left\{\operatorname{ABS}\left(R n^{j}-G^{j}\right)+\operatorname{ABS}\left(R n^{j+1}-G^{j+1}\right)\right\} \text { for } l E
\end{array}\right\} .
$$

Equation (9) and Equation (10) are nonlinear two element simultaneous equation. The two unknown variables can be estimated for the limit to which $\varepsilon$ is minimized, allowing $H$ and $I E$ to be estimated. Note that the other factors are obtained from observations or calculations independently using the aforementioned relationships.

\subsubsection{Initial Values for Ts and rehs}

Setting of the initial value of $T s$ and rehs are the most important. Figure 2 describes the concept of the initial values in the plane of vapor pressure and temperature 
$[e(T) \sim T]$ relationship. The dotted line indicates a partial vapor pressure for a given relative humidity. The saturated vapor pressure calculated by Tetens Formula.

On the plane, we can select two point as $a\left[(T z, e(T z)]\right.$ and $b\left[T_{0}, e(T z)\right]$ using observed data. If we assume rehs $=r e h z, c\left[T_{0}, e\left(T_{0}\right)\right]$ point can be determined, thus, the hatched triangle can be defined as the initial values. The ratio of $l E_{\text {est }} t H_{\text {est }}$ is similar to $[e(T s)-e(T z)] /(T s-T z)$ [refer Equation (2) or Equation (3) if $e(T)$ converted to $q(T)]$. We consider the optimized value of $b[T s, e(T z)]$ and $c[T s, e(T s)]$ as triangle with dotted line on the figure. We searched the optimal value $b[T s, e(T z)]$ and $c[T s, e(T s)]$ at the vicinity of the initial value $b\left[T_{0}, e(T z)\right]$ and $c\left[T_{0}, e\left(T_{0}\right)\right]$. Based on the above concept, we set the initial value of $T_{s}=T_{0}(R T s=0)$ and rehs $=$ rehz.

To guarantee better reproducibility, we set the constraints as Equation (13) as indicating in Figure 2 [2]:

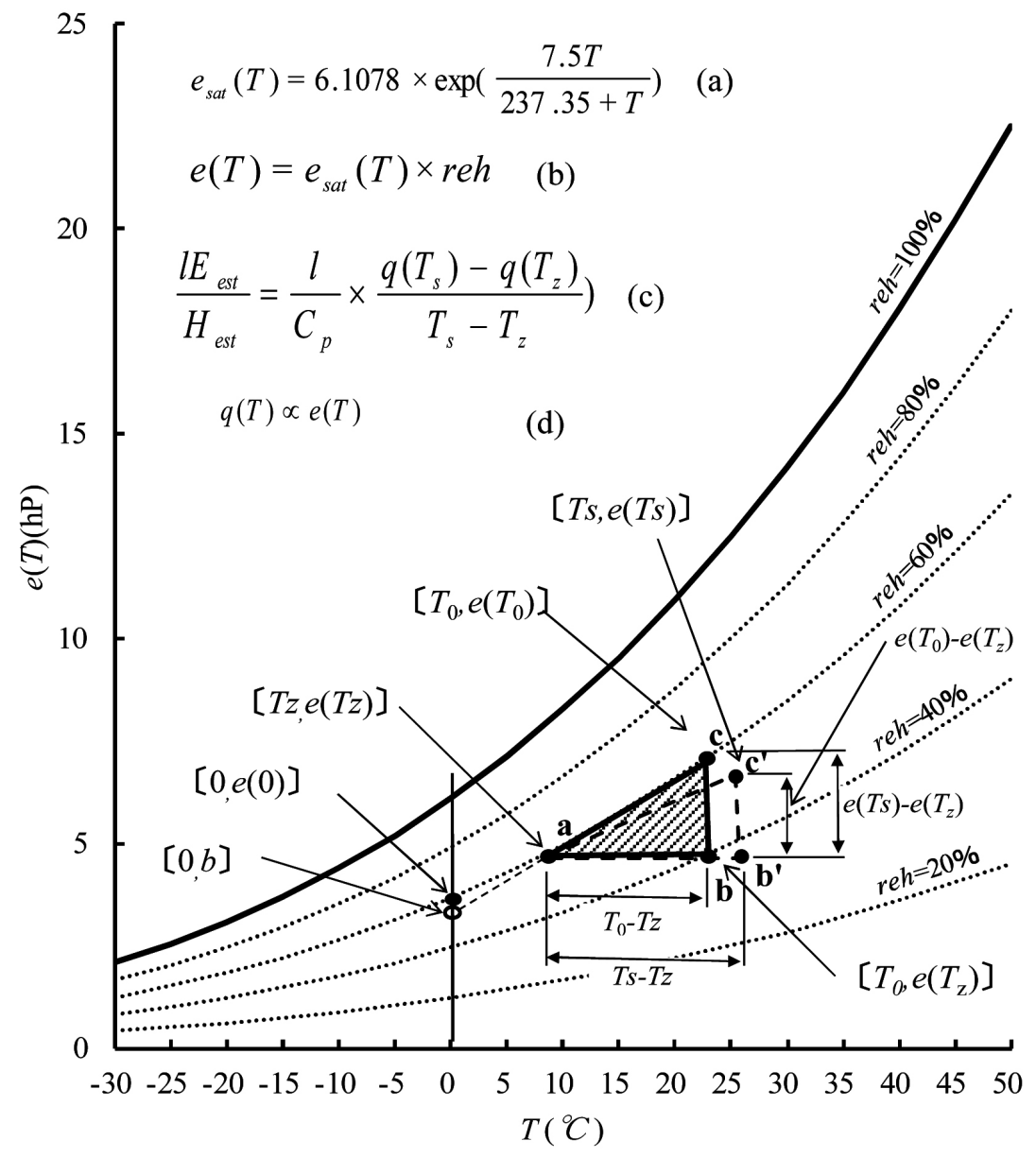

Figure 2. Schematic expression of initial value of $[e(T s)-e(T z)] /(T s-T z)$ on the $T \sim e(T)$ plane (The saturated vapor pressure $T \sim e_{s a t}(T)$-relationship obtained by Tetens Formula (a). The unsaturated (partial) vapor pressure $T \sim e(T)$-line calculated by multiplying relative humidity $(r e h) . I E_{\text {est }} / H_{\text {est }}$ is similar to the relationship $T s-T z$ over $[q(T s)-q(T z)]$ from Equation (c). Specific humidity $q(T)$ is proportional to vapor pressure $(T)$. Thus, the hatched triangle is similarity to $I E_{\text {est }} / H_{\text {est. }}$ The ratio $I E_{\text {est }} / H_{\text {est }}$ is set as the initial value of optimization. The dotted triangle is a schematic presentation of converged ratio of $l E_{e s t} / H_{\text {est }}$. The initial triangle point $c\left[T_{0}, e\left(T_{0}\right)\right]$ should be set as possible as the vicinity of the final triangle point $c[T s$, $e(T s)$ ]. That is very important things). 


$$
b=q(T s)-\frac{q(T s)-q(T z)}{T s-T z} \times T s .
$$

$b$ is a constant passing through a straight line at $T=0{ }^{\circ} \mathrm{C}$ with gradient $[q(T s)-q(T z)] /(T s-T z)$. The $b$ is experimental constant to compensate the weakness of sensitivity in the optimization process. The maximum of $b$ is defined as follows:

$$
b_{\max }=q_{\text {sat }}\left(T_{0}\right) \times r e h z .
$$

Using $b$ and $b_{\max }$, the constraint set as follows:

$$
0 \leq b \leq b_{\max } .
$$

\subsubsection{Actual Process of the Analysis}

The estimate of $H_{e s t}$ and $l E_{e s t}$ is performed as follows: First, the value of $T s$ is chosen from observed values $\left(T_{0}\right)$ collected near the soil surface. The rehs value is set to be the same as the rehz observed value. Second, the Ts and rehs are optimized together to satisfy the heat balance relationship using Equation (9) and Equation (10).

The values of $\varepsilon_{i}$ are initially very small, on the order of $10^{-15}\left(\mathrm{~W} \cdot \mathrm{m}^{-2}\right)$. Therefore, the objective function is multiplied by $10^{15}$. To avoid abnormal fluctuations of $H_{\text {est }}$ and $I E_{e s t}$ constraints on those variables are set as less than ABS $(R n-G)$. The constraints for $B_{a p p}$ are set at $-100<B_{a p p}<100$ by referring to the singularity of convergence process [1] [2] and actual data. And also applied the constraint as $0<b<b_{\max }$. The reason for these constrains is described in the discussion section.

The calculation follows a non-linear optimization procedure that employs a General Reduced Gradient (GRG) algorithm, which can be applied with the Excel Solver on a personal computer (Appendix 1 and Appendix 2) [1] [2].

\subsection{Investigation Site and Equipment}

To examine the proposed method, eight sites were chosen throughout the world as listed in Table 1: three sites are in the USA and Australia and one site is in Europe and China. The data of all sites were prepared by FLUXNET (Beringer, et al. [7]: Sturt Plains (AU-Stp) Ozflux L2 Data; Billesbach, et al. [8]: Woodward (US-AR2) Data; Ceschia and Tallec [9]: Lamasquere (FR-Lam) Data; Eamus and Cleverly [10]: Ti Tree East (AU-TTE) Ozflux L2 Data; Beringer J. and L. Hutley [11]: Dry River (AU-Dry) Ozflux L2 Data; Scott [12]: Lucky Hills Shrubland (US-Whs) AmeriFlux L2 Data; Wharton [13]: Diablo (US-Dia) AmeriFlux L2 Data; Yanhong, et al. [14]: Qinghai Flux Research Site (CN-QHB) AsiaFlux Data).

Table 1 shows the name of the sites, country, state/province, location, elevation, vegetation, tower height, plant height and year of examined data. The tested year ware chosen as well as minimize the data gaps.

Table 2 describes the type of instruments applied with the variables of the heat balance components, unit and description of those measurements, including temperature $T_{0}$ near the soil surface at depth $d_{T 0} \mathrm{~cm}$.

To investigate the accuracy of the observed data, Table 3 describes the heat balance relationship of observed at the tested sites that expressed in the heat flux. The 
Table 1. Feature of the tested sites (all sites active core measurement presently being made).

\begin{tabular}{|c|c|c|c|c|c|c|c|c|}
\hline 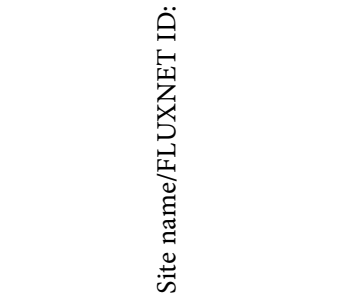 & 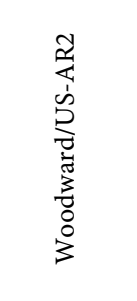 & 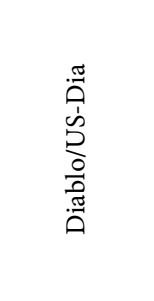 & 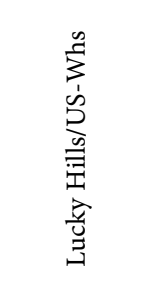 & 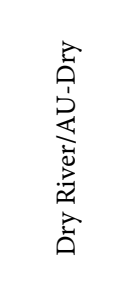 & 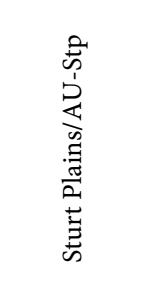 & 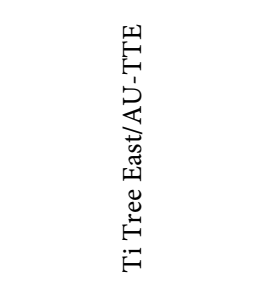 & 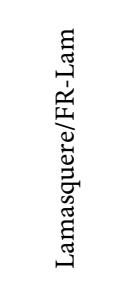 & 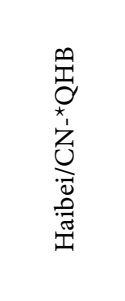 \\
\hline Country: & USA & USA & USA & Australia & Australia & Australia & France & China \\
\hline State/Province: & Oklahoma & California & Arizona & $\begin{array}{l}\text { Northern } \\
\text { Territory }\end{array}$ & $\begin{array}{l}\text { Northern } \\
\text { Territory }\end{array}$ & Northern Territory & - & $\begin{array}{c}\text { Qinghai, } \\
\text { China }\end{array}$ \\
\hline Latitude (+: N/-: S): & 36.6358 & 37.6773 & 31.7438 & -15.2588 & -17.1507 & -22.287 & 43.4965 & 37.6 \\
\hline Longitude (+: E/-: W): & -99.5975 & -121.5296 & -110.0522 & 132.3706 & 133.3502 & 133.64 & 1.2379 & 101.3333 \\
\hline Elevation: & $646 \mathrm{~m}$ & $323 \mathrm{~m}$ & $1372 \mathrm{~m}$ & $175 \mathrm{~m}$ & $250 \mathrm{~m}$ & $600 \mathrm{~m}$ & $182 \mathrm{~m}$ & $3250 \mathrm{~m}$ \\
\hline Vegetation (IGBP): & Grasslands & Grasslands & $\begin{array}{c}\text { Open } \\
\text { shrublands }\end{array}$ & $\begin{array}{l}\text { Woodland } \\
\text { savanna }\end{array}$ & $\begin{array}{c}\text { Mitchell } \\
\text { Grasslands }\end{array}$ & $\begin{array}{c}\text { Open } \\
\text { Corymbia-hummock } \\
\text { savanna and Mulga } \\
\text { patches }\end{array}$ & Cropland & $\begin{array}{l}\text { Alpine } \\
\text { meadow }\end{array}$ \\
\hline Tower height: & - & about $3.0 \mathrm{~m}$ & about $6.5 \mathrm{~m}$ & $\begin{array}{c}\text { abuot } \\
15 \mathrm{~m}\end{array}$ & about $5 \mathrm{~m}$ & about $10 \mathrm{~m}$ & - & about $3 \mathrm{~m}$ \\
\hline Plant height: & - & $<1.0 \mathrm{~m}$ & - & $12.3 \mathrm{~m}$ & $0.5 \mathrm{~m}$ & $4.85 \mathrm{~m}$ & - & 0.3 \\
\hline Data available & 2010 & 2012 & 2014 & 2012 & 2014 & 2013 & 2008 & 2004 \\
\hline (period) & $1 / 1-12 / 31$ & $1 / 1-12 / 31$ & $1 / 1-12 / 31$ & $1 / 1-12 / 31$ & $1 / 1-12 / 31$ & $1 / 1-12 / 31$ & $1 / 1-12 / 31$ & $1 / 1-12 / 31$ \\
\hline
\end{tabular}

Table 2. Measurement instruments of the tested sites including $d_{70}$.

\begin{tabular}{|c|c|c|c|c|c|c|c|c|c|}
\hline & 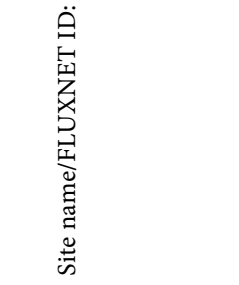 & 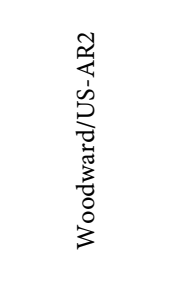 & 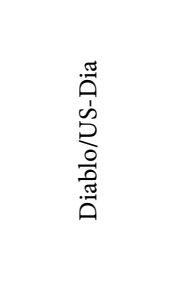 & 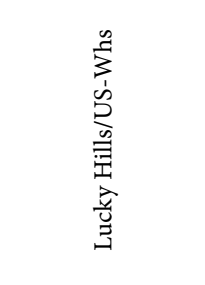 & 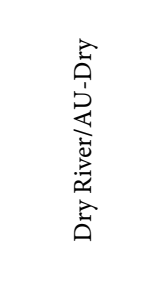 & 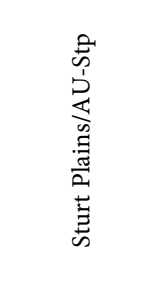 & 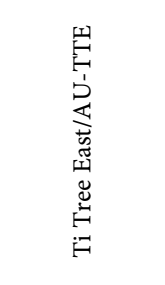 & 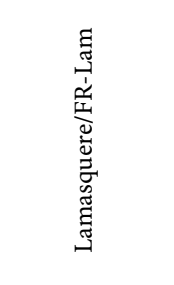 & 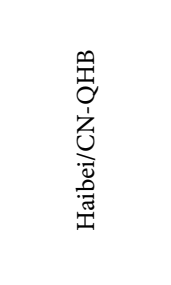 \\
\hline Variable & Units Description & Model & Model & Model & Model & Model & Model & Model & Model \\
\hline$F G$ & $\mathrm{~W} \cdot \mathrm{m}^{-2}$ Soil heat flux & $\begin{array}{c}\text { Soil Heat Flux } \\
\text { Plate } \\
\text { (HFT-3.1, } \\
\text { Radiation and } \\
\text { Energy } \\
\text { Balance } \\
\text { Systems) }\end{array}$ & $\begin{array}{c}\text { Soil Heat Flux } \\
\text { Plate } \\
\text { (HFT-3-L, } \\
\text { Campbell) }\end{array}$ & $\begin{array}{c}\text { Soil Heat } \\
\text { Flux Plate } \\
\text { (REBS Inc., WA) }\end{array}$ & $\begin{array}{l}\text { Soil heat } \\
\text { flux (HFT3, } \\
\text { Campbell) }\end{array}$ & $\begin{array}{l}\text { Soil heat } \\
\text { flux (HFT3, } \\
\text { Campbell) }\end{array}$ & $\begin{array}{l}\text { Ground heat } \\
\quad \text { flux } \\
\text { (CN3, } \\
\text { Middleton) }\end{array}$ & $\begin{array}{l}\text { Soil Heat Flux } \\
\text { Plate } \\
\text { (HFP-01, } \\
\text { Hukseflux) }\end{array}$ & $\begin{array}{c}\text { Heat flux plate } \\
\text { (HFT-3, } \\
\text { Campbell) }\end{array}$ \\
\hline$H$ & $\begin{array}{c}\mathrm{W} \cdot \mathrm{m}^{-2} \text { Sensible heat } \\
\text { flux }\end{array}$ & $\begin{array}{c}\text { Sonic } \\
\text { Anemometer } \\
\text { (WindMaster } \\
\text { PRO, Gill) }\end{array}$ & $\begin{array}{c}\text { Sonic } \\
\text { Anemometer } \\
\text { (CSAT3A, } \\
\text { Campbell) }\end{array}$ & $\begin{array}{c}\text { Sonic } \\
\text { Anemometer } \\
\text { (CSAT3, } \\
\text { Campbell) }\end{array}$ & $\begin{array}{c}\text { Sonic } \\
\text { Anemometer } \\
\text { (CSAT3, } \\
\text { Campbell) }\end{array}$ & $\begin{array}{c}\text { Sonic } \\
\text { Anemometer } \\
\text { (CSAT3, } \\
\text { Campbell) }\end{array}$ & $\begin{array}{c}\text { Sonic } \\
\text { Anemometer } \\
\text { (CSAT3, } \\
\text { Campbell) }\end{array}$ & $\begin{array}{c}\text { Sonic } \\
\text { Anemometer } \\
\text { (CSAT3, } \\
\text { Campbell) }\end{array}$ & $\begin{array}{c}\text { Sonic } \\
\text { anemometer } \\
\text { (CSAT-3, } \\
\text { Campbell) }\end{array}$ \\
\hline
\end{tabular}




\begin{tabular}{|c|c|c|c|c|c|c|c|c|c|c|}
\hline$L E$ & $\mathrm{~W} \cdot \mathrm{m}^{-2}$ & $\begin{array}{l}\text { Latent heat } \\
\text { flux }\end{array}$ & $\begin{array}{c}\text { Sonic } \\
\text { Anemometer } \\
\text { (WindMaster } \\
\text { PRO, Gill), } \\
\text { Open Path } \\
\mathrm{CO}_{2} / \mathrm{H}_{2} \mathrm{O} \\
\text { Gas Analyzer } \\
\text { (LI-7500, } \\
\text { LI-COR) }\end{array}$ & $\begin{array}{c}\text { Sonic } \\
\text { Anemometer } \\
\text { (CSAT3A, } \\
\text { Campbell), } \\
\text { Open Path } \\
\mathrm{CO}_{2} / \mathrm{H}_{2} \mathrm{O} \\
\text { Gas Analyzer } \\
\text { (EC150, } \\
\text { Campbell) }\end{array}$ & $\begin{array}{c}\text { Sonic } \\
\text { Anemometer } \\
\text { (CSAT3, } \\
\text { Campbell), } \\
\text { Open Path } \\
\mathrm{CO}_{2} / \mathrm{H}_{2} \mathrm{O} \\
\text { Gas Analyzer } \\
\text { (LI-7500, } \\
\text { LI-COR) }\end{array}$ & $\begin{array}{c}\text { Sonic } \\
\text { Anemometer } \\
\text { (CSAT3, } \\
\text { Campbell), } \\
\text { Infrared Gas } \\
\text { Analyser } \\
\text { (LI-7500, } \\
\text { Li-cor) }\end{array}$ & $\begin{array}{c}\text { Sonic } \\
\text { Anemometer } \\
\text { (CSAT3, } \\
\text { Campbell), } \\
\text { Open Path } \\
\mathrm{CO}_{2} / \mathrm{H}_{2} \mathrm{O} \\
\text { Gas Analyzer } \\
\text { (LI-7500, } \\
\text { LI-COR) }\end{array}$ & $\begin{array}{c}\text { Sonic } \\
\text { Anemometer } \\
\text { (CSAT3, } \\
\text { Campbell), } \\
\text { Open Path } \\
\mathrm{CO}_{2} / \mathrm{H}_{2} \mathrm{O} \\
\text { Gas Analyzer } \\
\text { (LI-7500A, } \\
\text { LI-COR) }\end{array}$ & $\begin{array}{c}\text { Sonic } \\
\text { Anemometer } \\
\text { (CSAT3, } \\
\text { Campbell), } \\
\text { Infrared Gas } \\
\text { Analyzer } \\
\text { (Li-7500, } \\
\text { Li-COR) }\end{array}$ & $\begin{array}{c}\text { Sonic } \\
\text { anemometer } \\
\text { (CSAT-3, } \\
\text { Campbell), } \\
\text { Open-path } \\
\mathrm{CO}_{2} / \mathrm{H}_{2} \mathrm{O} \\
\text { Gas Analyzer } \\
\text { (LI-7500, } \\
\text { LI-COR) }\end{array}$ \\
\hline PREC & $\mathrm{mm}$ & Precipitation & $\begin{array}{c}\text { Tipping } \\
\text { Bucket } \\
\text { Rain Gauge } \\
\text { (TE-525, } \\
\text { Texas } \\
\text { Electronics) }\end{array}$ & $\begin{array}{l}\text { Tipping Bucket } \\
\text { Rain Gauge } \\
(260-2500-12, \\
\text { NovaLynx Co.) }\end{array}$ & $\begin{array}{l}\text { Tipping Bucket } \\
\text { Rain Gauge } \\
\text { (TE525, camp- } \\
\text { bell) }\end{array}$ & $\begin{array}{l}\text { Rain gauge } \\
\text { (CS702, } \\
\text { Campbell) }\end{array}$ & $\begin{array}{l}\text { Rain gauge } \\
\text { (CS702, } \\
\text { Campbell) }\end{array}$ & $\begin{array}{l}\text { Rain gauge } \\
\text { (CS700, } \\
\text { Hydrological } \\
\text { Services ) }\end{array}$ & $\begin{array}{l}\text { Tipping } \\
\text { Bucket } \\
\text { Rain gauge } \\
\text { (ARG100, } \\
\text { Campbell) }\end{array}$ & $\begin{array}{l}\text { Tipping bucket } \\
\text { raingage } \\
\text { (TE525MM, } \\
\text { CSL) }\end{array}$ \\
\hline PRESS & $\mathrm{kPa}$ & $\begin{array}{l}\text { Barometric } \\
\text { pressure }\end{array}$ & $\begin{array}{l}\text { Barometric } \\
\text { Pressure } \\
\text { Sensor } \\
\text { (PTB101, } \\
\text { Vaisala) }\end{array}$ & $\begin{array}{c}\text { Open Path } \\
\mathrm{CO}_{2} / \mathrm{H}_{2} \mathrm{O} \\
\text { Gas Analyzer } \\
\text { (EC150, } \\
\text { Campbell) }\end{array}$ & $\begin{array}{c}\text { Open Path } \\
\mathrm{CO}_{2} / \mathrm{H}_{2} \mathrm{O} \\
\text { Gas Analyzer } \\
\text { (LI-7500, } \\
\text { LI-COR) }\end{array}$ & $\begin{array}{c}\text { Infrared Gas } \\
\text { Analyser } \\
\text { (LI7500, Licor) }\end{array}$ & $\begin{array}{c}\text { Open Path } \\
\mathrm{CO}_{2} / \mathrm{H}_{2} \mathrm{O} \\
\text { Gas Analyzer } \\
\text { (LI-7500A, } \\
\text { LI-COR) }\end{array}$ & $\begin{array}{c}\text { Barometric } \\
\text { Pressure Sensor } \\
\text { (CS106, Vaisala) }\end{array}$ & $\begin{array}{l}\text { Infrared Gas } \\
\text { Analyzer } \\
\text { (Li-7500, } \\
\text { Licor) }\end{array}$ & $\begin{array}{c}\text { Open-path } \\
\mathrm{CO}_{2} / \mathrm{H}_{2} \mathrm{O} \text { Gas } \\
\text { Analyzer } \\
\text { (LI-7500, } \\
\text { LI-COR) }\end{array}$ \\
\hline RH & $\%$ & $\begin{array}{l}\text { Relative } \\
\text { humidity of } \\
\text { air }\end{array}$ & $\begin{array}{c}\text { Temperature/ } \\
\text { Humidity } \\
\text { Probe } \\
\text { (HMP50Y, } \\
\text { Vaisala) }\end{array}$ & $\begin{array}{c}\text { Temperature/ } \\
\text { Humidity } \\
\text { Probe } \\
\text { (HMP45, } \\
\text { Vaisala) }\end{array}$ & $\begin{array}{c}\text { Temperature/ } \\
\text { Humidity Probe } \\
\text { (HMP45, } \\
\text { Vaisala) }\end{array}$ & $\begin{array}{c}\text { Temperature/ } \\
\text { Humidity } \\
\text { Probe } \\
\text { (HMP45C, } \\
\text { Vaisala) }\end{array}$ & $\begin{array}{c}\text { Temperature/ } \\
\text { Humidity Probe } \\
\text { (HMP45C, } \\
\text { Vaisala) }\end{array}$ & $\begin{array}{c}\text { Temperature/ } \\
\text { Humidity Probe } \\
\text { (HMP45C, } \\
\text { Vaisala) }\end{array}$ & $\begin{array}{l}\text { Temperature/ } \\
\text { Humidity } \\
\text { Probe } \\
\text { (HMP35A, } \\
\text { Vaisala) }\end{array}$ & $\begin{array}{c}\text { Temperature/ } \\
\text { Humidity Probe } \\
\text { (HMP45C, } \\
\text { Vaisala) }\end{array}$ \\
\hline$R n$ & $\mathrm{~W} \cdot \mathrm{m}^{-2}$ & $\begin{array}{c}\text { Net } \\
\text { radiation }\end{array}$ & $\begin{array}{c}\text { Net } \\
\text { Radiometer } \\
\text { (NR-LITE-L, } \\
\text { Kipp \& } \\
\text { Zonen) }\end{array}$ & $\begin{array}{c}\text { Net Radiometer } \\
\text { (NR-LITE-L, } \\
\text { Kipp \& Zonen) }\end{array}$ & $\begin{array}{c}\text { Net Radiometer } \\
\text { (CNR2, Kipp \& } \\
\text { Zonen) }\end{array}$ & $\begin{array}{l}\text { Net Radiometer } \\
\text { (NR-LITE, } \\
\text { Kipp \& Zonen) }\end{array}$ & $\begin{array}{c}\text { Net Radiometer } \\
\text { (NR-LITE, Kipp } \\
\text { \& Zonen) }\end{array}$ & $\begin{array}{c}\text { Net Radiometer } \\
\text { (CNR1, Kipp \& } \\
\text { Zonen) }\end{array}$ & $\begin{array}{c}\text { Net } \\
\text { Radiometer } \\
\text { (CNR1, Kipp } \\
\text { \& Zonen) }\end{array}$ & $\begin{array}{c}\text { Net Radiometer } \\
\text { (CNR1, Kipp \& } \\
\text { Zonen) }\end{array}$ \\
\hline$T A$ & $\operatorname{deg} C$ & $\begin{array}{c}\text { Air } \\
\text { temperature }\end{array}$ & $\begin{array}{c}\text { Temperature/ } \\
\text { Humidity } \\
\text { Probe } \\
\text { (HMP50Y, } \\
\text { Vaisala) }\end{array}$ & $\begin{array}{c}\text { Temperature/ } \\
\text { Humidity } \\
\text { Probe } \\
\text { (HMP45, } \\
\text { Vaisala) }\end{array}$ & $\begin{array}{c}\text { Temperature/ } \\
\text { Humidity Probe } \\
\text { (HMP45, } \\
\text { Vaisala) }\end{array}$ & $\begin{array}{c}\text { Temperature/ } \\
\text { Humidity } \\
\text { Probe } \\
\text { (HMP45C, } \\
\text { Vaisala) }\end{array}$ & $\begin{array}{c}\text { Temperature/ } \\
\text { Humidity Probe } \\
\text { (HMP45C, } \\
\text { Vaisala) }\end{array}$ & $\begin{array}{c}\text { Temperature/ } \\
\text { Humidity Probe } \\
\text { (HMP45C, } \\
\text { Vaisala) }\end{array}$ & $\begin{array}{l}\text { Temperature/ } \\
\text { Humidity } \\
\text { Probe } \\
\text { (HMP35A, } \\
\text { Vaisala) }\end{array}$ & $\begin{array}{c}\text { Temperature/ } \\
\text { Humidity Probe } \\
\text { (HMP45C, } \\
\text { Vaisala) }\end{array}$ \\
\hline$T_{0}$ & $\operatorname{deg} C$ & $\begin{array}{c}\text { Soil } \\
\text { temperature }\end{array}$ & $\begin{array}{c}\text { Thermocouples } \\
\text { (Type E) }\end{array}$ & $\begin{array}{c}\text { Soil } \\
\text { Thermocouple } \\
\text { Probe } \\
\text { (TCAV-L, } \\
\text { Campbell) }\end{array}$ & Thermocouples & $\begin{array}{c}\text { Soil } \\
\text { Thermocouple } \\
\text { Probe } \\
\text { (TCAV, } \\
\text { Campbell) }\end{array}$ & $\begin{array}{c}\text { Soil } \\
\text { Thermocouple } \\
\text { Probe } \\
\text { (TCAV, } \\
\text { Campbell ) }\end{array}$ & $\begin{array}{c}\text { Soil } \\
\text { Thermocouple } \\
\text { Probe } \\
\text { (TCAV, } \\
\text { Campbell ) }\end{array}$ & $\begin{array}{c}\text { Soil } \\
\text { Temperature } \\
\text { Probe } \\
(107, \\
\text { Campbell })\end{array}$ & $\begin{array}{c}\text { Thermocouple } \\
\text { (Copper- } \\
\text { constantan) }\end{array}$ \\
\hline$d T_{0}$ & $\mathrm{~cm}$ & $\begin{array}{c}\text { Depth of } \\
\text { measurement }\end{array}$ & 5 & 5 & 5 & 5 & 8 & 10 & 5 & 5 \\
\hline
\end{tabular}

imbalance was estimated by $\operatorname{Im} b=R n-G-l E-H$ using observed annual data and the imbalance ratio defined as $R a_{i m b}=\operatorname{Imb} /(R n-G)$. There are numerous data gaps at all sites on an hourly basis. 


\section{Result}

\subsection{Conversion of Observed Data $\left(H_{o b s}\right.$ and $\left.l E_{o b s}\right)$ into Corrected Data ( $H_{\text {cor }}$ and $I E_{\text {cor }}$ )}

The observed data do not achieve the heat balance relationship shown in Table 3. To maintain the heat balance, multiple regression analysis is applied using Equation (8) as aforementioned. Figure 3 describes the relationship $(R n-G)$ versus $(H+I E)$ of the original and corrected data at all tested sites. The observed data are shown in the red circle, whereas the corrected data are shown in the blue circle. The slope of the corrected data at the tested sites mostly increased and approached 1.0 [1] [2].

The regression coefficients for all tested sites are described in Table 4 that $A$ is for $I E$ and $B$ is for $H$. The correction coefficient $A$ ranged from 0.836 to 1.495 for $H$, with an average of 1.130, and the coefficient $B$ ranged from 0.950 to 1.312 for $H$, with an average of 1.157. The observed data are corrected using these coefficients for all tested sites. The imbalance ratio $R a i_{m b}$ before and after correction is described in Table 3.

Table 3. Annual heat balance relationship of the tested sites (except no observed data): Data gap, one of which $G, T z, T_{0}, P($ atmospheric pressure), rehz, $R n, H_{o b s}$ and $l E_{o b s}$, is do not use for analysis. Imbalance is calculated by $\operatorname{Imb}=R n-G-l E-H$ using yearly observed data and the imbalance ratio defined as $R a_{i m b}=\operatorname{Imb} /(R n-G)$.

\begin{tabular}{|c|c|c|c|c|c|c|c|c|c|}
\hline \multirow{2}{*}{ Site name } & $R n$ & $G$ & $H$ & $L E$ & Imbalance & Raimb & & Data gap & Precipitation \\
\hline & $\mathrm{W} \cdot \mathrm{m}^{-2}$ & $\mathrm{~W} \cdot \mathrm{m}^{-2}$ & $\mathrm{~W} \cdot \mathrm{m}^{-2}$ & $\mathrm{~W} \cdot \mathrm{m}^{-2}$ & $\mathrm{~W} \cdot \mathrm{m}^{-2}$ & Before & After & $\%$ & $\mathrm{~mm} \cdot \mathrm{year}^{-1}$ \\
\hline US-Ur2 & 25,940 & -87 & 16,659 & 12,964 & -3596 & -0.138 & 0.006 & 5 & 463 \\
\hline US-Dia & 30,714 & 157 & 17,286 & 6374 & 6897 & 0.226 & 0.033 & 7 & 297 \\
\hline US-Whs & 35,942 & 369 & 23,183 & 9449 & 2941 & 0.083 & -0.011 & 9 & 339 \\
\hline AU-Dry & 40,093 & 1704 & 17,144 & 18,552 & 2693 & 0.070 & 0.023 & 31 & 664 \\
\hline AU-Stp & 41,607 & 917 & 24,942 & 16,599 & -850 & -0.021 & -0.007 & 18 & 899 \\
\hline AU-TTE & 37,548 & 1086 & 29,694 & 3716 & 3053 & 0.084 & 0.128 & 35 & 180 \\
\hline FR-Lam & 22,299 & -673 & 5473 & 11,242 & 6256 & 0.272 & 0.169 & 35 & 785 \\
\hline $\mathrm{CN}-\mathrm{QHB}$ & 31,081 & -367 & 10,363 & 13,045 & 8040 & 0.256 & 0.053 & 16 & 601 \\
\hline
\end{tabular}

Table 4. Regression coefficients $A$ for $I E$ and $B$ for $H$ : Regression coefficient is the same with correction coefficient of observed $H$ and $I E$.

\begin{tabular}{|c|c|c|c|}
\hline Site Name & A & B & $\mathrm{R}^{2}$ \\
\hline US-AR2 & 1.093 & 1.178 & 0.991 \\
\hline US-Dia & 1.333 & 1.160 & 0.981 \\
\hline US-Whs & 0.836 & 0.950 & 0.970 \\
\hline AU-Dry & 1.229 & 1.083 & 0.987 \\
\hline AU-Stp & 1.038 & 1.134 & 0.980 \\
\hline AU-TTE & 0.978 & 1.151 & 0.976 \\
\hline FR-Lam & 1.037 & 1.284 & 0.852 \\
\hline $\mathrm{CN}-\mathrm{QHB}$ & 1.495 & 1.312 & 0.976 \\
\hline Average & 1.130 & 1.157 & 0.964 \\
\hline
\end{tabular}




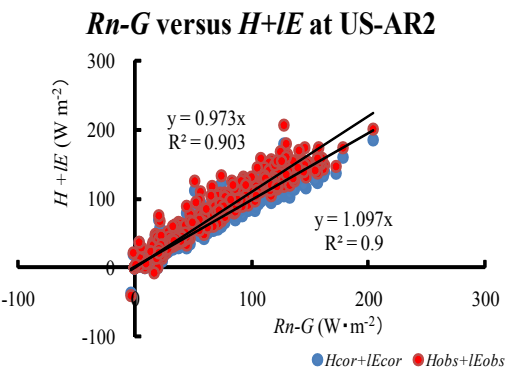

$R n-G$ versus $H+l E$ at AU-Dry

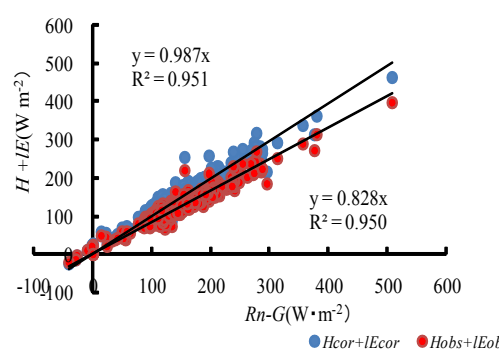

HcortlEcor $\bullet$ HobstlEobs

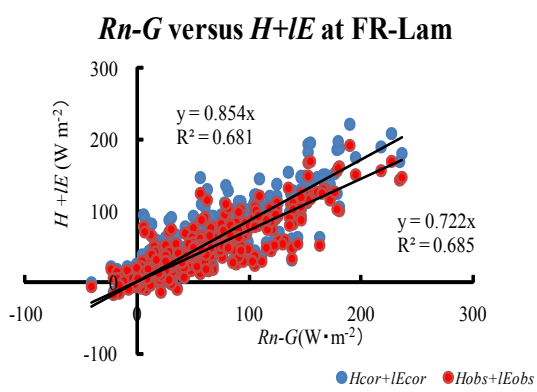

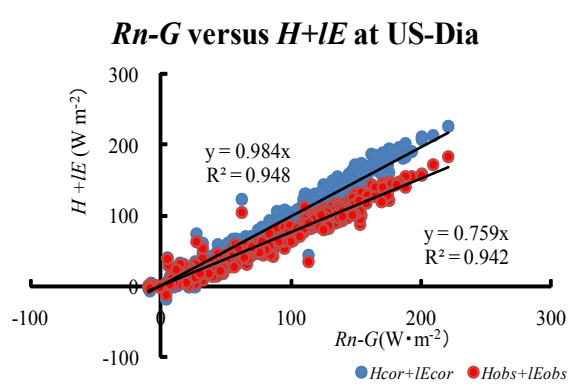

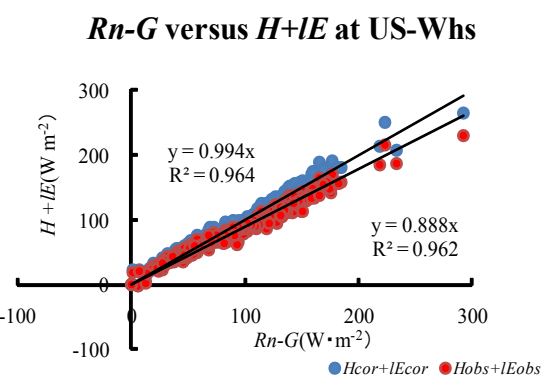

$R n-G$ versus $H+l E$ at AU-Stp
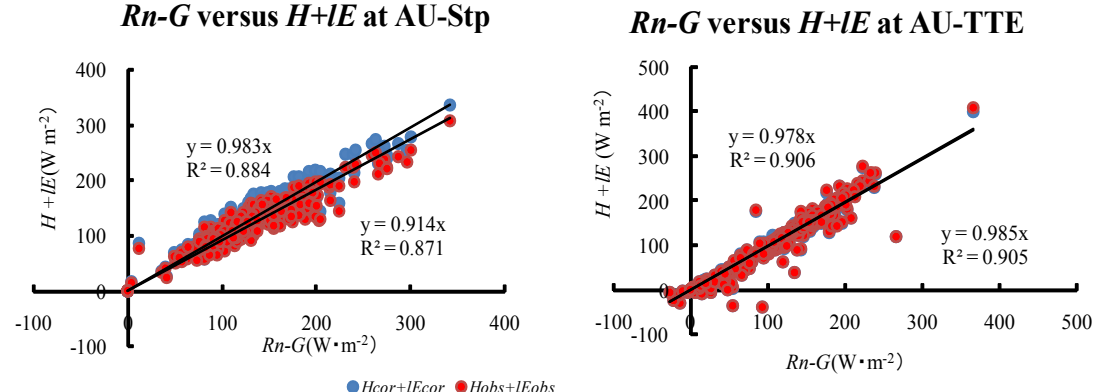

-Hcor+lEcor Hobs+lEobs

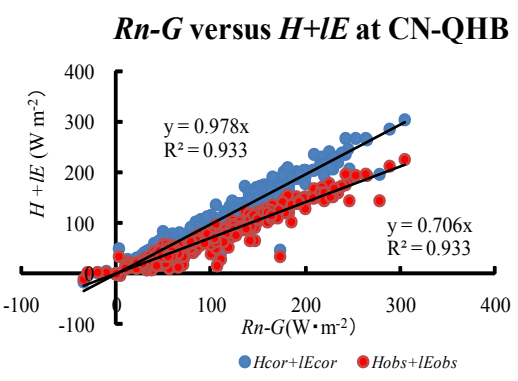

Figure 3. Relationship between $(R n-G)$ versus $(H+I E)\left(\mathrm{W} \cdot \mathrm{m}^{-2}\right)$ : According to the heat balance relationship, $R n+G=H+I E$ must be guaranteed by actual data. Therefore, $R n+G$ versus $H+I E$ compared. But $R n+G$ does not coincide with $H+I E$ as red circle in the figure, to improve the relationship, regression analysis performed. The blue circle indicates the result of the regression analysis at eight tested sites.

\subsection{Result of the General Solution by Equation (9) and Equation (10)}

We set the initial value as $T s=T_{0}$ and rehs $=$ rehz based on the triangle concept in Figure 2 as aforementioned. According to the initial values, the heat balance relationship is initially almost satisfied. The constraints Equation (15) applied for all sites except FRLam and CN-QHB because these two sites having relatively large amount of precipitation. This reason describe in the discussion section.

The estimation of $T s$ and rehs is conducted with precision $=0.000001$ and convergence $=0.0001$ in Solver option. In addition, heat balance is not achieved instantaneously; it requires a few hours [3]. Thus, the figure adjusts to a five-hour moving average. The comparison procedure is similar to the previous research [1] [2].

\subsection{Comparison of the Hourly Change of $I E$ and $H$ Estimated and Corrected}

To confirm the validity of the general method, Figure 4 compares the hourly change in $I E_{\text {cor }}$ with $I E_{\text {est }}$ and $H_{\text {cor }}$ with $H_{\text {est }}$ at all sites in summer. All sites are very well consisted with each other. However, in detail, $I E_{\text {est }}$ is not consistent with $I E_{c o r}$ at US-Dia and $H_{\text {est }}$ is 
$I E$ at US-AR2 (2010)

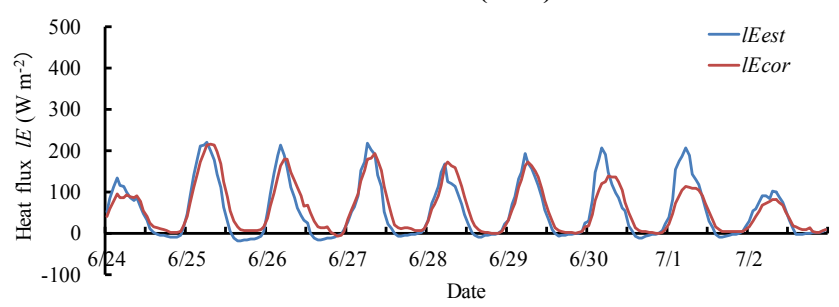

IE at US-Dia (2012)

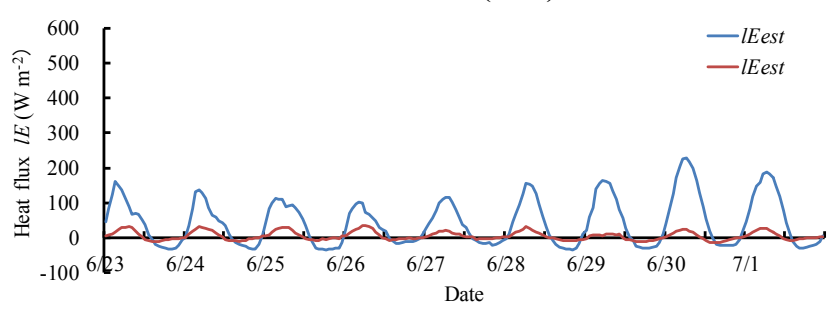

$I E$ at US-Whs (2010)

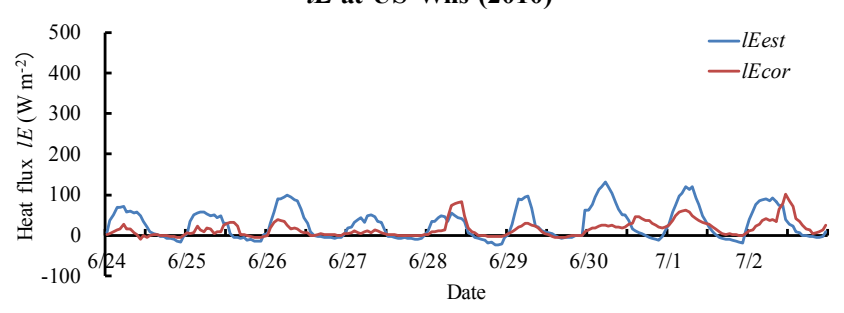

$I E$ at AU-Dry (2012)

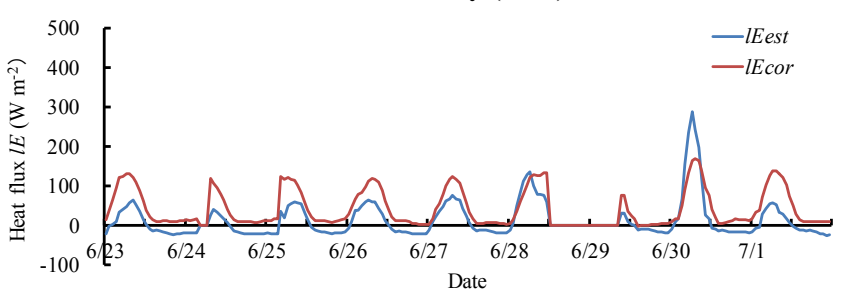

IE at AU-Stp (2014)

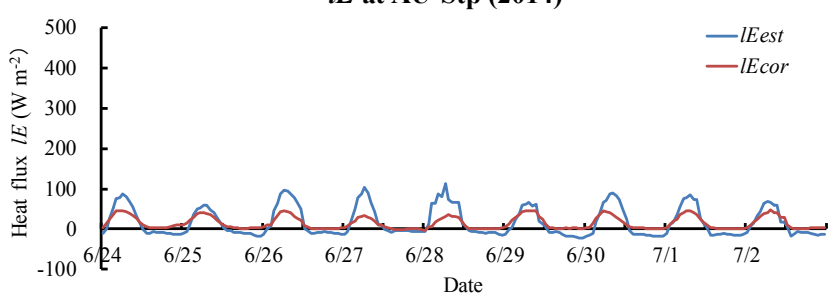

IE at AU-TTE (2013)

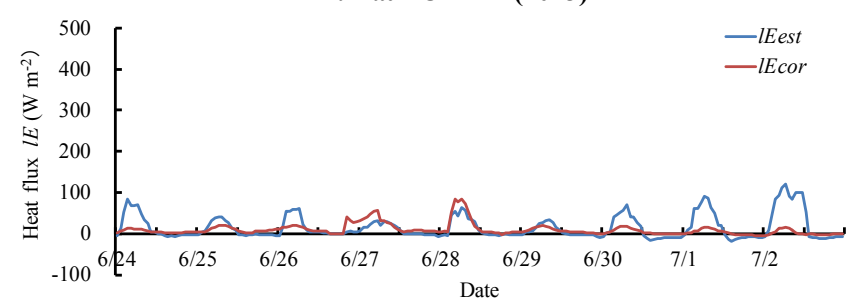

$H$ at US-AR2 (2010)

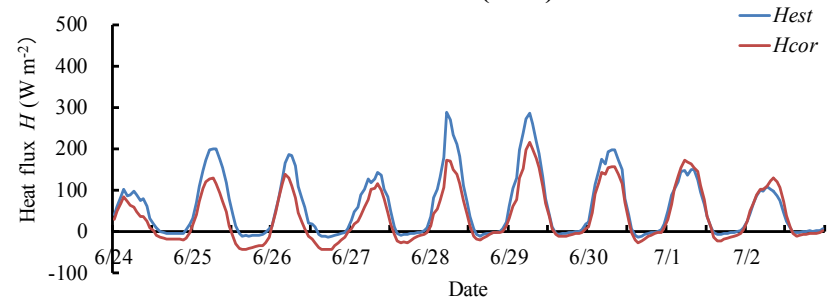

$H$ at US-Dia (2012)

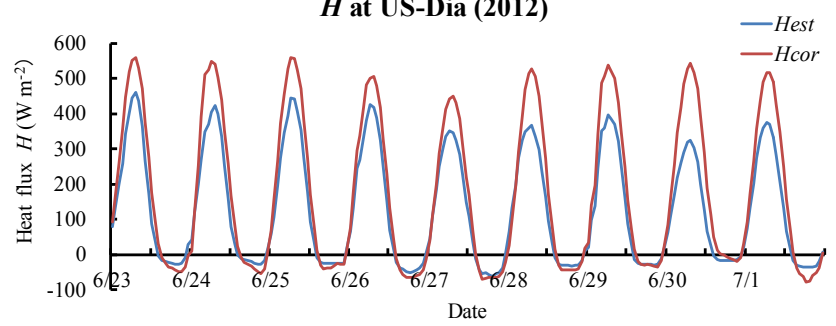

$H$ at US-Whs (2010)

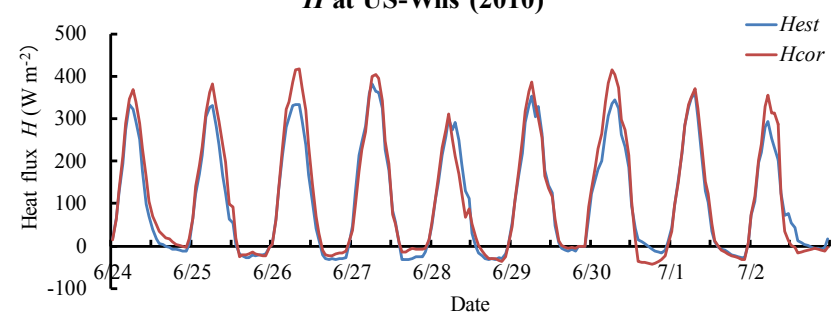

$H$ at AU-Dry (2012)

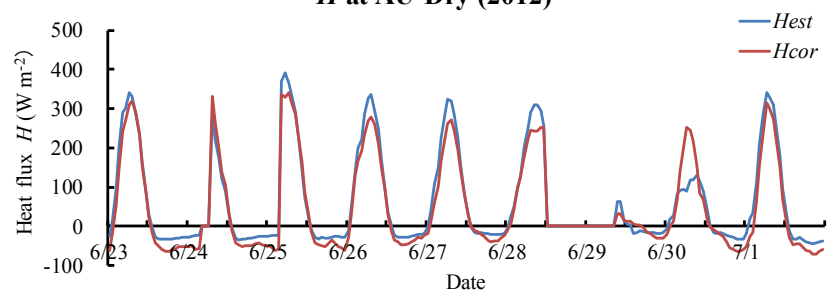

$H$ at AU-Stp (2014)

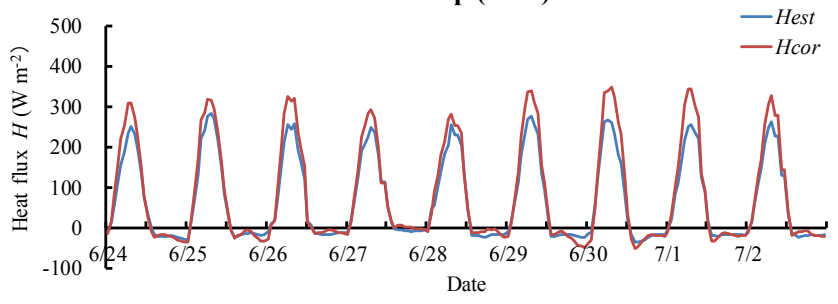

$H$ at AU-TTE (2013)

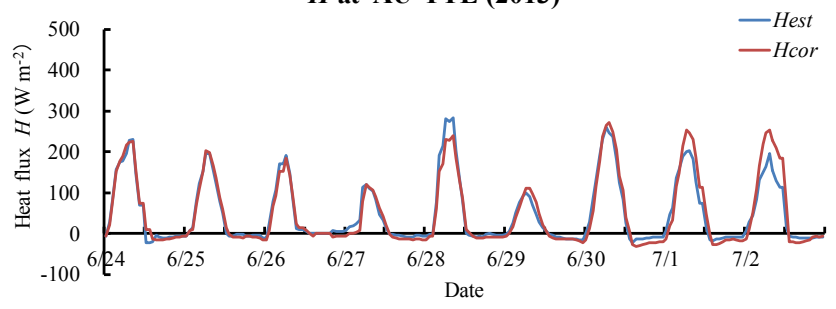




\section{$l E$ at FR-Lam (2008)}

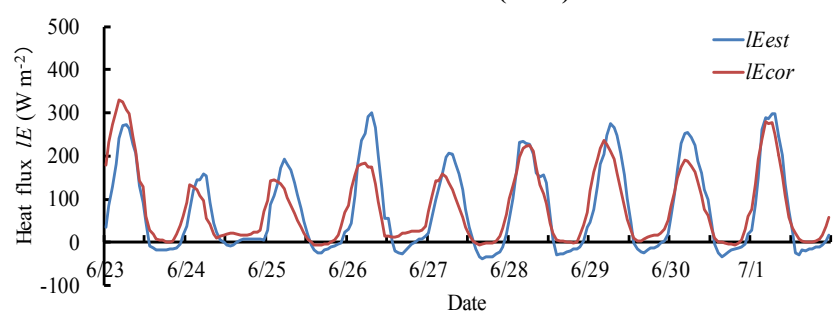

$l E$ at CN-QHB (2004)

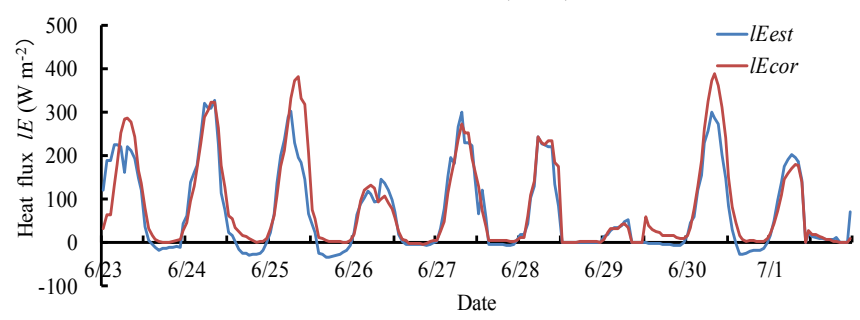

$H$ at FR-Lam (2008)

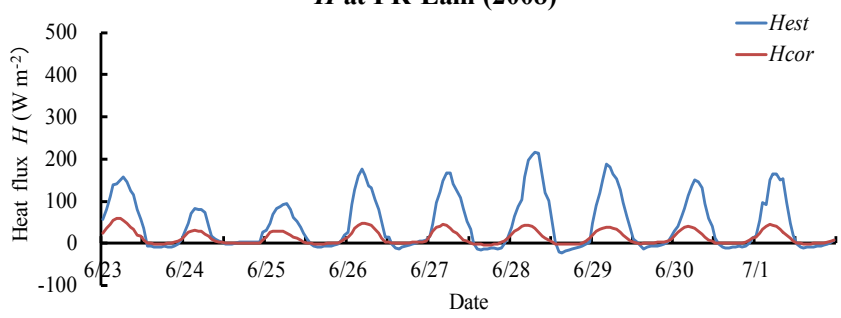

$\mathrm{H}$ at CN-QHB (2004)

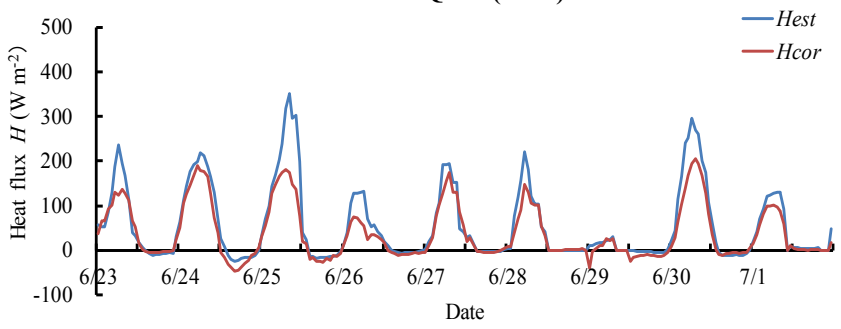

Figure 4. Hourly change of $I E$ and $H$ observed and estimated at the tested sites by general method $(1)\left(\mathrm{W} \cdot \mathrm{m}^{-2}\right)$ : The left hand sites describe the comparison of estimated and corrected $I E$ and right hand side describe the estimated and corrected $H$ at all tested sites.

not consistent with $H_{\text {cor }}$ at FR-Lam. In the former case, $I E_{\text {est }}$ is overestimated, while $H_{\text {est }}$ is underestimated. The other items, such as $I E_{o b s}$ and $I E_{i m b}$, indicate almost similar trends, and $H_{o b s}$ and $H_{i m b}$ also show the same trend but with small differences (not shown).

\subsection{Annual Change of the Estimated and Observed $I E$ and $H$}

To investigate annual change in the estimated and observed $I E$ and $H$, Figure 5 describes the changes of those variables throughout the year. All sites describe the trend is relatively well reproduced.

However, in detail, at AU-Dry and AU-Stp, $I E_{\text {est }}$ indicate underestimate at former part of the year while $H_{\text {est }}$ the overestimate at the same period. At FR-Lam, $I E_{\text {est }}$ indicate a little underestimate at the year while $H_{\text {est }}$ are a little overestimate. The other items of $I E_{o b s}$ and $I E_{i m b}$ exhibit similar trends, and $H_{o b s}$ and $H_{i m b}$ also display the same trend but with small differences (not shown).

\subsection{Comparison of the Observed and Estimated $I E$ and $H$}

To confirm the validity of the general solution (1), Figure 6 compares the observed versus estimated data of $I E$ and $H$ on a daily basis. If the slope (slope of the straight line) is 1.0 , the observed value coincides well with the estimated value. For $H$, the slopes of the observed versus estimated relationship nearly coincide $(<15 \%)$ at the all sites except AU-Dry. For $H$, all sites indicate underestimate $(>15 \%) . R^{2}$ values (determination coefficient) shows a small values $(<60 \%)$ for all sites except US-Dia $(0.815)$ and AUTTE (0.822), US-Whs (0.745). The other items of $I E_{o b s}$ and $I E_{i m b}$ are exhibited similar trends, and $H_{o b s}$ and $H_{i m b}$ show the same trend but with small differences (not shown). In addition, boundary of estimation accuracy is selected as $15 \%$ by referring the heat imbalance of original data. 
$l E$ at US-AR2 (2010)

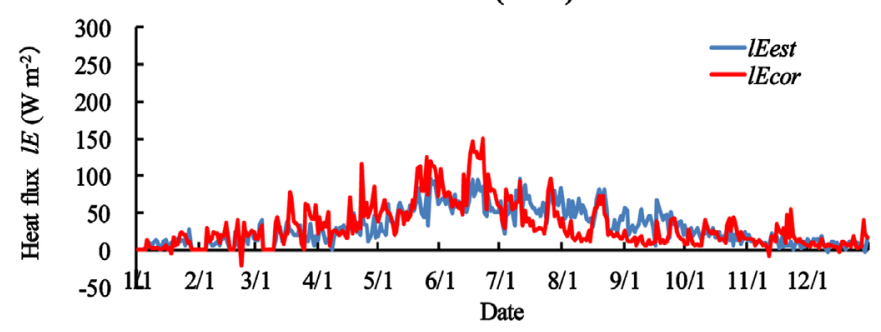

$l E$ at US-Dia (2012)

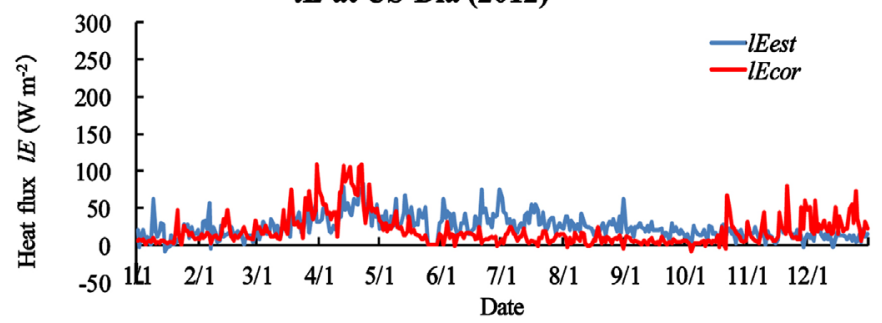

$l E$ at US-Whs (2010)

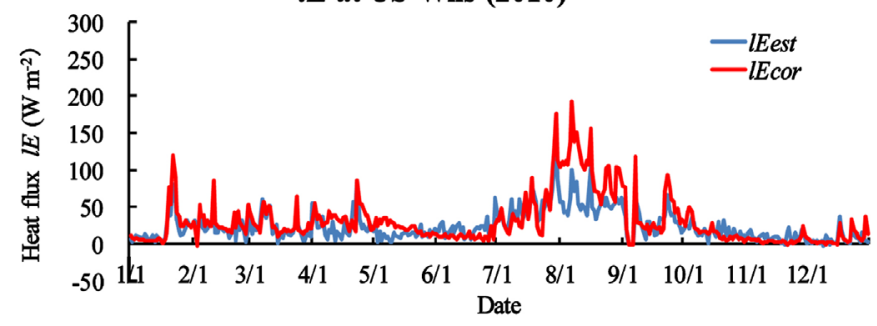

lE at AU-Dry (2012)

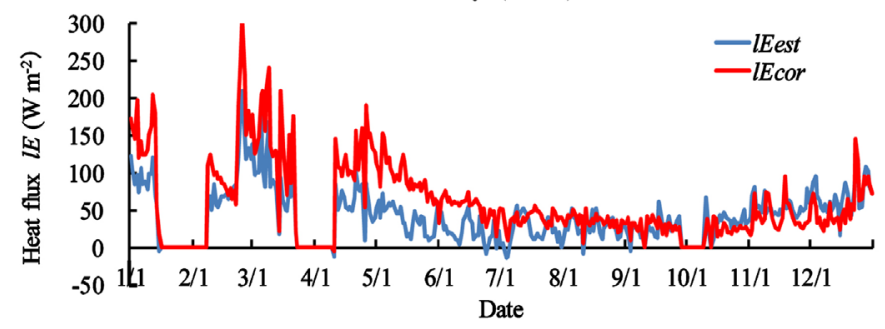

IE at AU-Stp(2014)

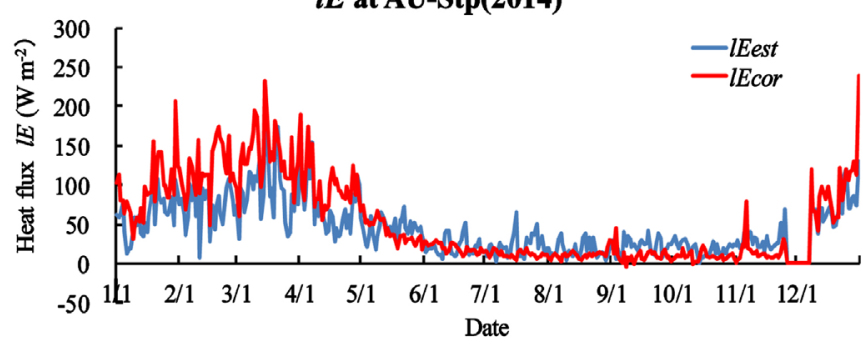

IE at AU-TTE (2013)

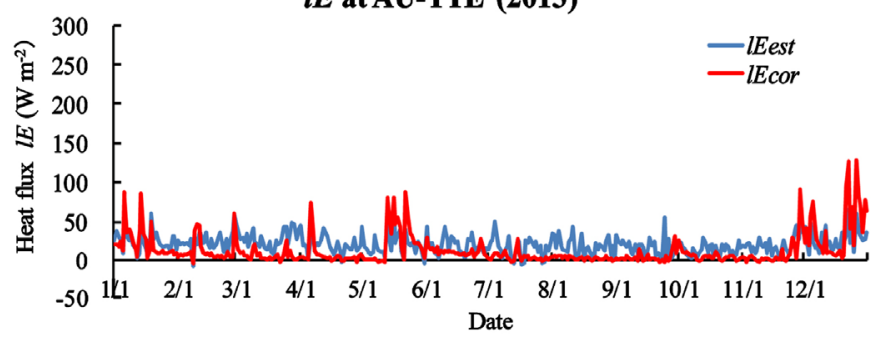

$H$ at US-Ar2 (2010)

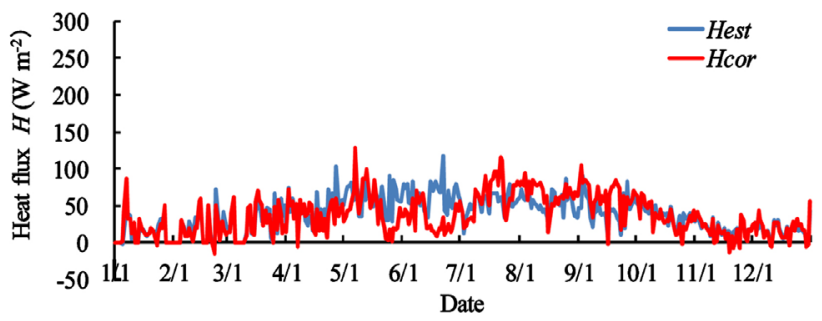

$H$ at US-Dia (2012)

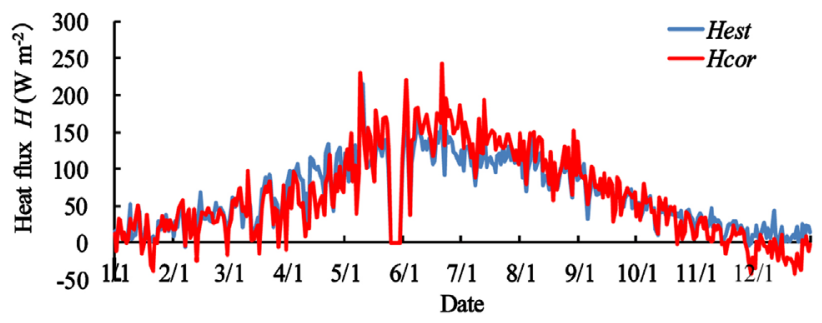

$H$ at US-Whs(2010)

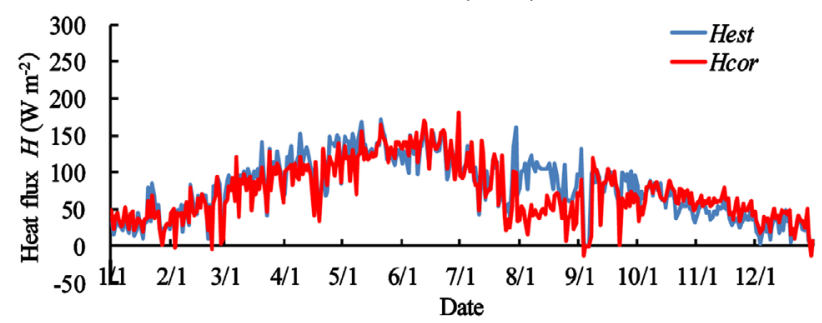

$H$ at AU-Dry (2012)

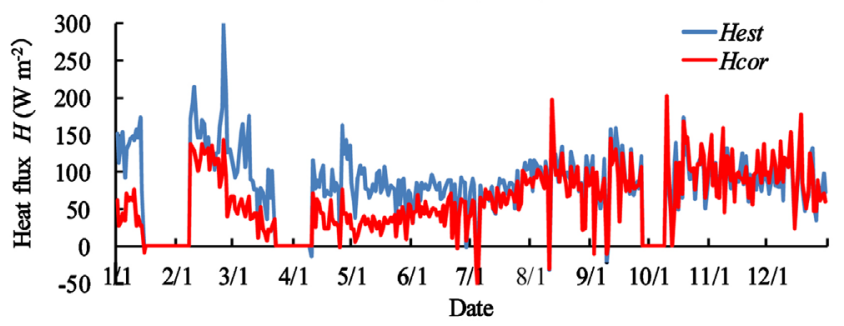

$H$ at AU-Stp(2014)

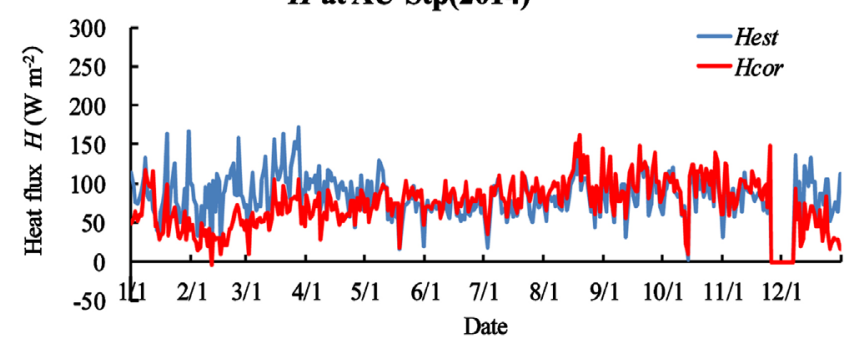

$H$ at AU-TTE (2013) $\quad-$ Hest

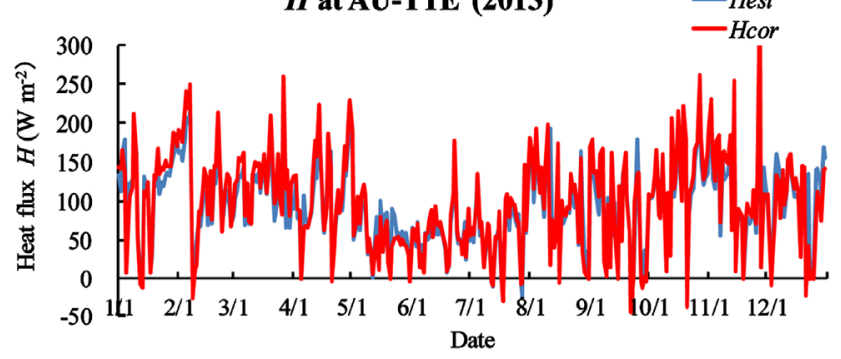


$l E$ at FR-Lam (2008)

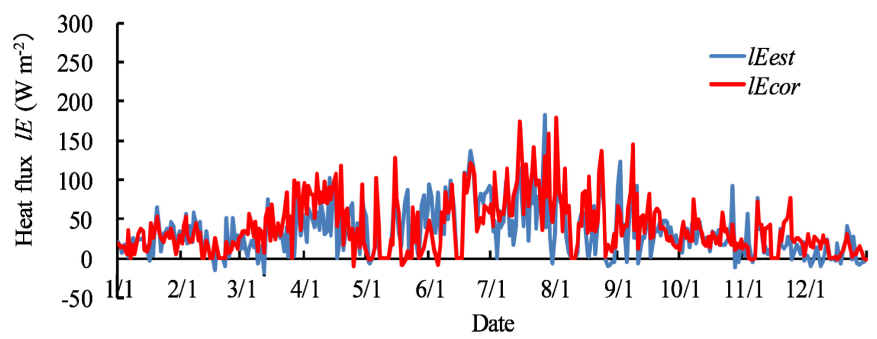

lE at CN-QHB (2004)

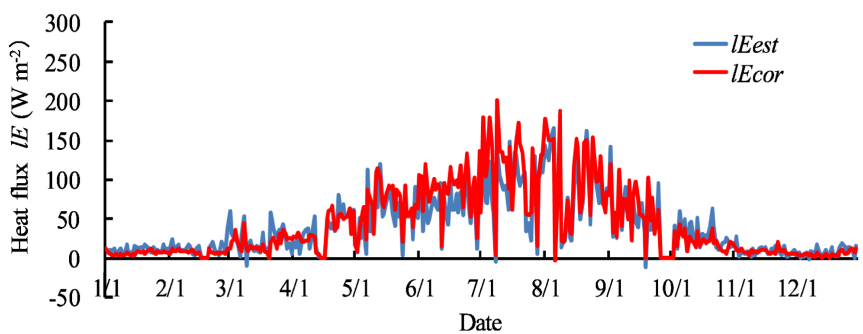

$H$ at FR-Lam (2008)

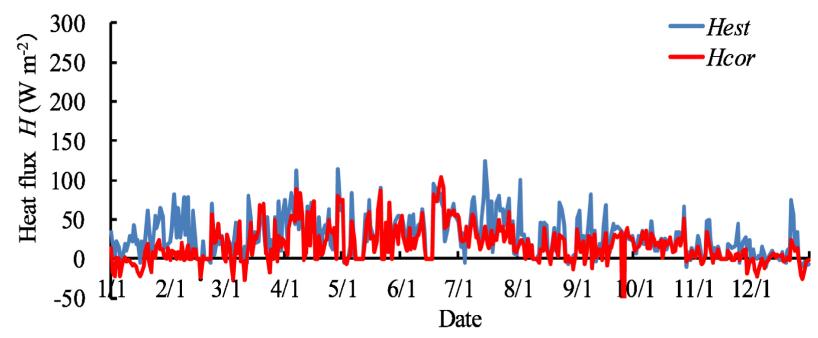

$H$ at CN-QHB (2004)

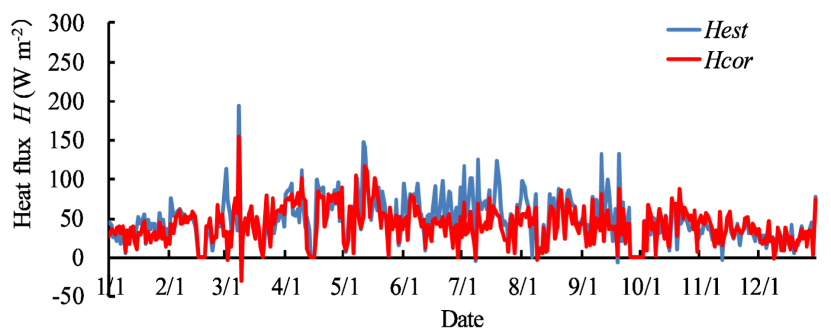

Figure 5. Annual change of $I E$ and $H$ estimated and observed by general method (1) $\left(\mathrm{W} \cdot \mathrm{m}^{-2}\right)$ : The left hand sites describe the comparison of estimated and corrected $I E$ and right hand side describe the estimated and corrected $H$ at all tested sites.

\subsection{Comparison of the Observed rehz with Estimated rehs and $T_{0}$ with Ts}

The relationship between the estimated rehs and the observed rehz, i.e., the initial values and converged values, is of great concern. The left hand side of Figure 7 describes the hourly changes of those two items in summer. The parameters exhibit quite similar trends, i.e., the difference between rehs and rehz is quite small. The right hand side of Figure 7 describes the change of $T s-T_{0}$ and $T s-T z . T_{0}$ is the observed temperature near the soil surface, and $T z$ is the air temperature, as previously mentioned. $T s-T_{0}$ changed periodically with daily changes about $\pm 3^{\circ} \mathrm{C}$ with site specific but sometimes shows a large difference. The difference between $T s-T z$ is approximately within $8^{\circ} \mathrm{C}$.

The above trend of rehs and $T s$ changes is quite similar in the other seasons and at the other sites, although small differences are observed (not shown). In addition, the discontinuous portion is originated from data gap.

\subsection{Comparison of Estimated and Observed Evapotranspiration Rate $(E T a)$}

Using observed and estimated $I E$, monthly evapotranspiration was obtained at the all sites, as shown in Figure 8 by assuming $100 \mathrm{~W} \cdot \mathrm{m}^{-2}$ equivalents for $3.53 \mathrm{~mm} \cdot \mathrm{day}^{-1}$ [15]. The initial value of rehse quals rehz and constrains as Equation (12) and Equation (15) was applied. In addition, if there are data gap in a given month, the average ETa in a day multiplied the number of days of the month.

All sites describe satisfactory well reproduced $H a$ and Eta except AU-Dry. In detail, although there are small differences between $H a_{o b s}, H a_{c o r}$ and $H a_{e s t,}$ and also ETa $a_{o b s} E T a_{c o n}$, and $E T a_{e s t}$ at all sites, the difference was relatively small. 


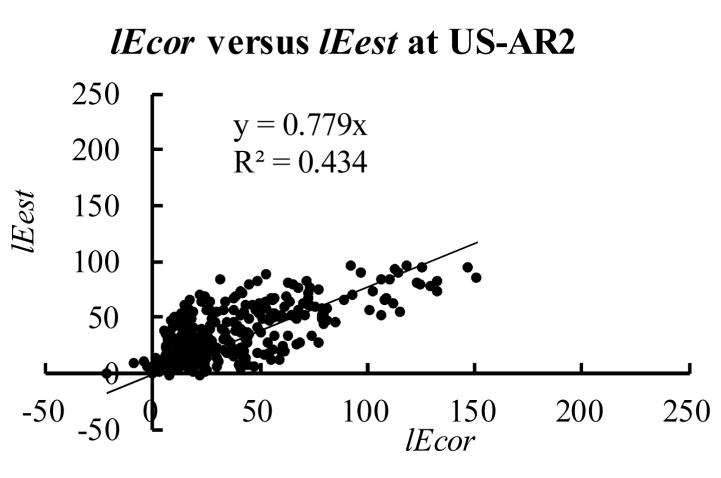

IEcor versus $l E$ Eest at US-Dia
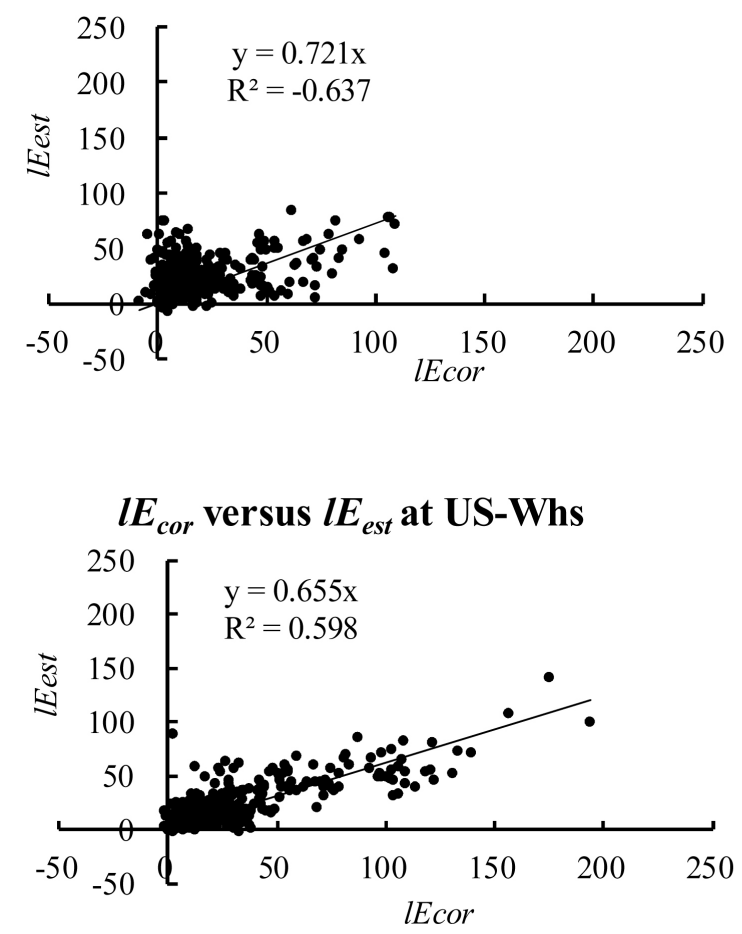

IEcor versus lEest at AU-Dry

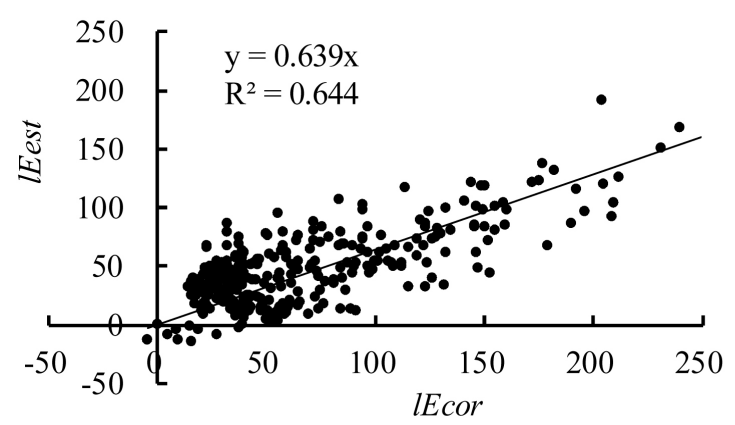

Hcor versus Hest at US-AR2
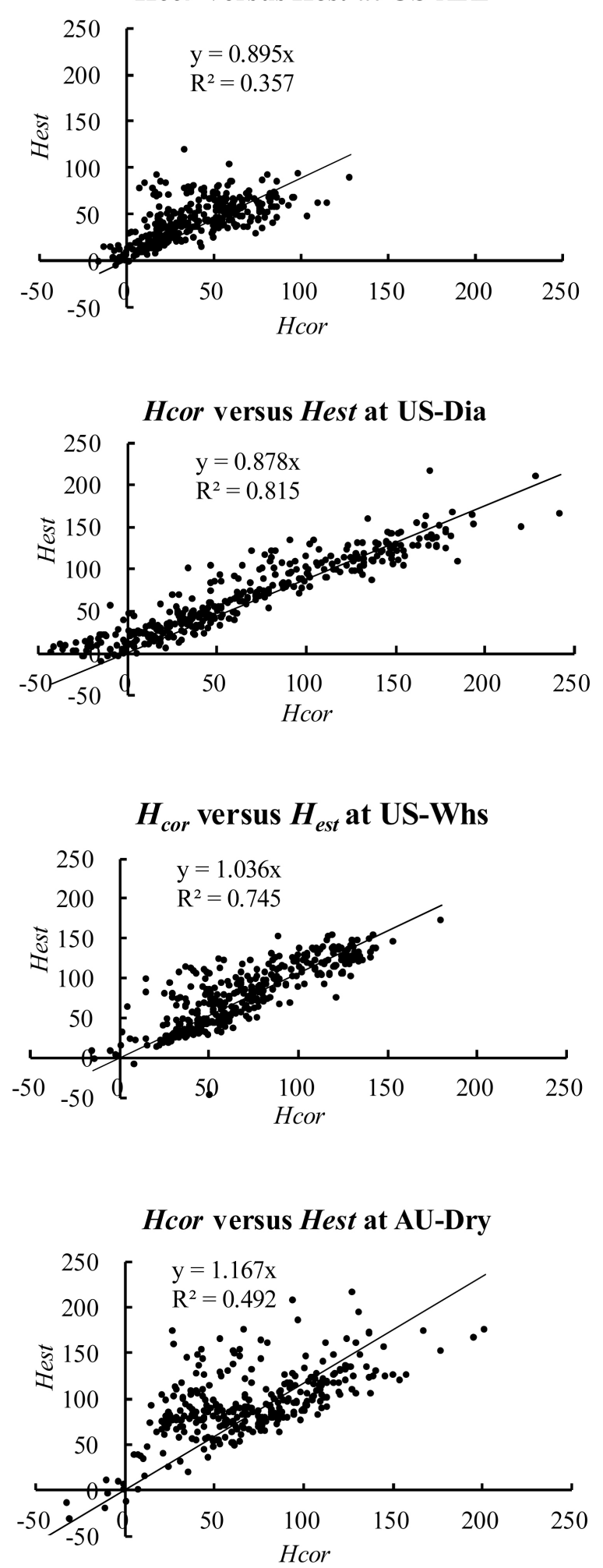
IEcor versus lEest at AU-Stp

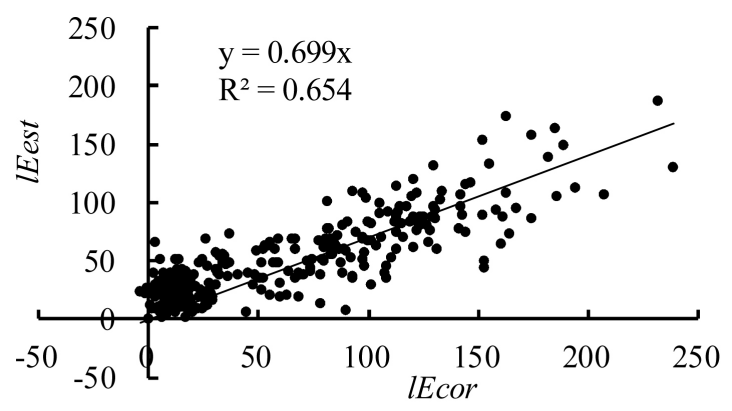

IEcor versus lEest at AU-TTE

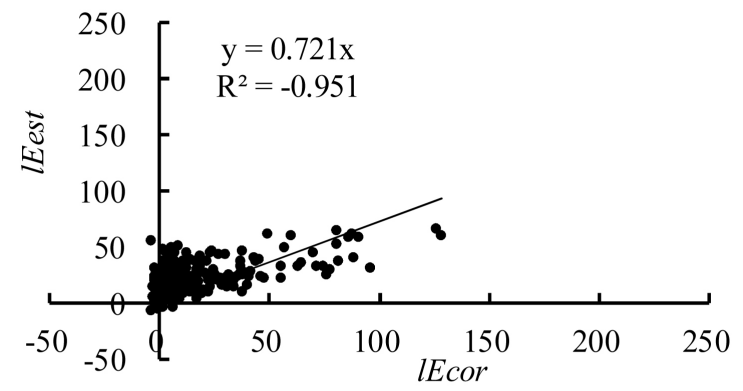

IEcor versus lEest at FR-Lam

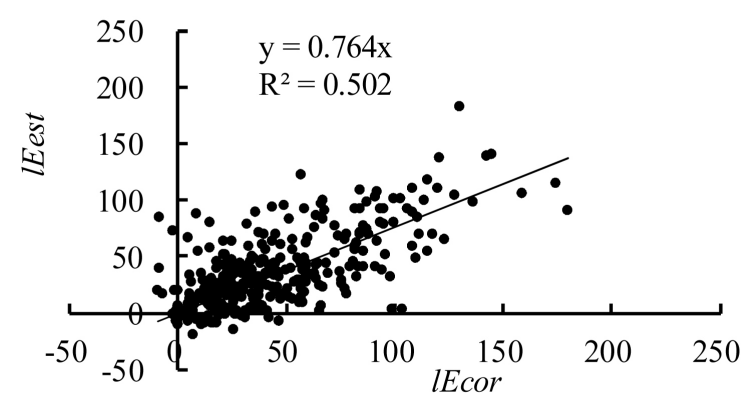

IEcor versus lEest at CN-QHB

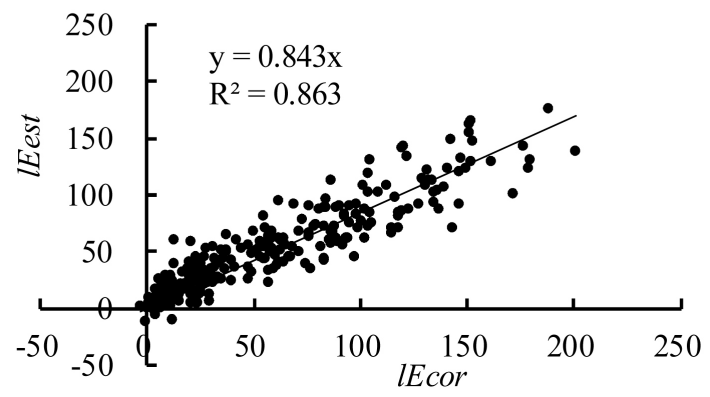

Hcor versus Hest at AU-Stp

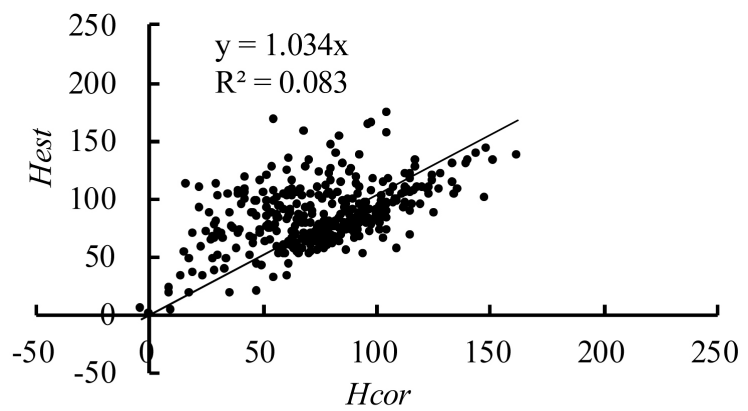

Hcor versus Hest at AU-TTE

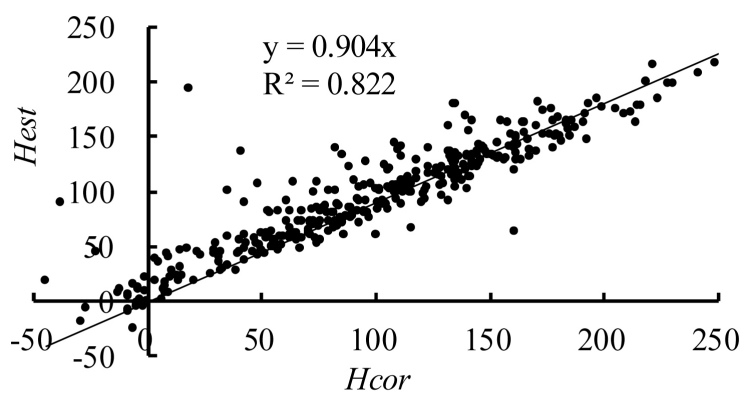

Hcor versus Hest at FR-Lam

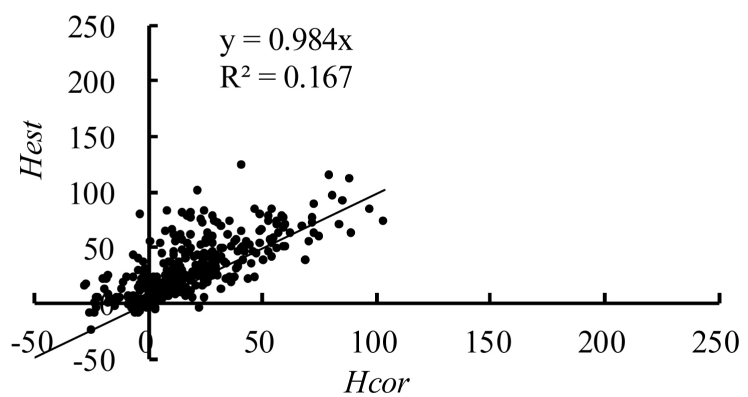

Hcor versus Hest at $\mathrm{CN}-\mathrm{QHB}$

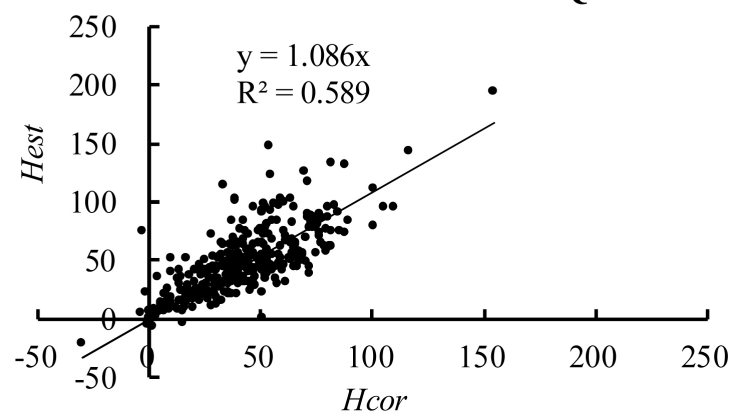

Figure 6. Comparison of $I E$ and $H$ estimated and observed at the tested sites by general method (1) $\left(\mathrm{W} \cdot \mathrm{m}^{-2}\right)$. 

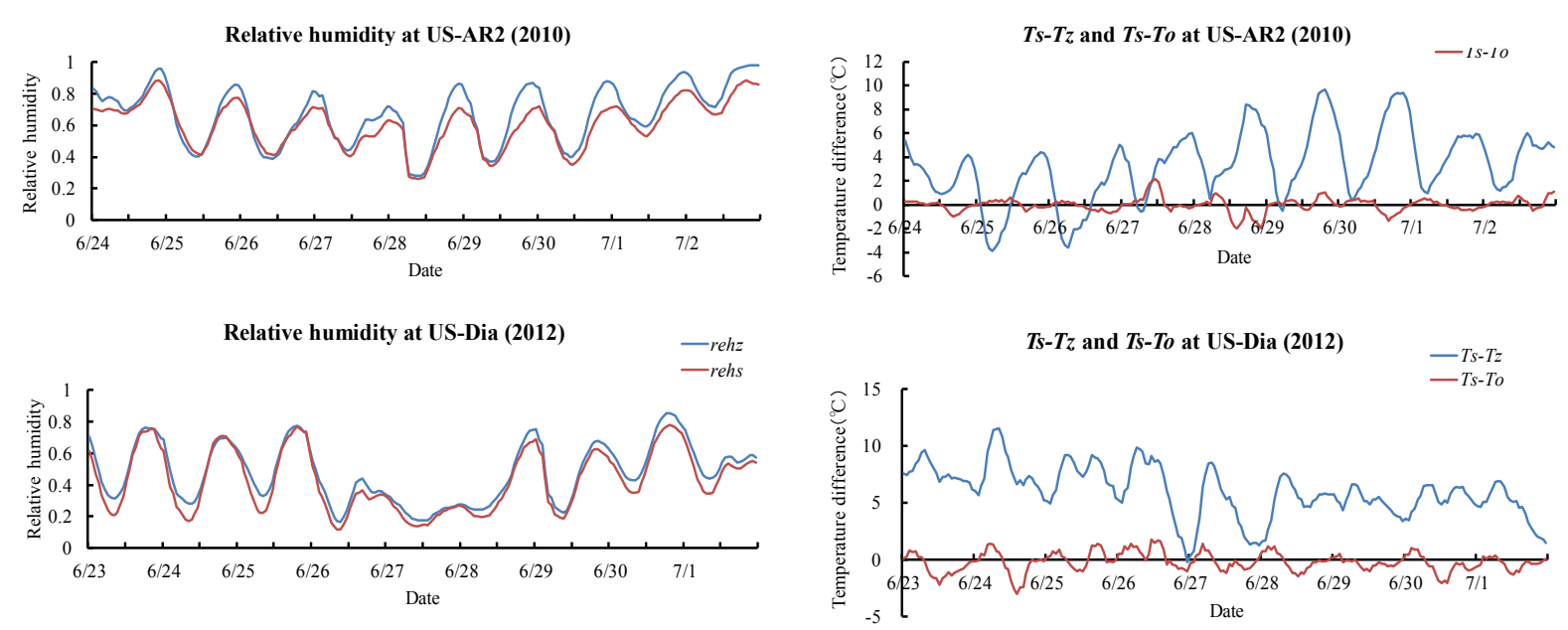

Relative humidity at US-Whs (2010)
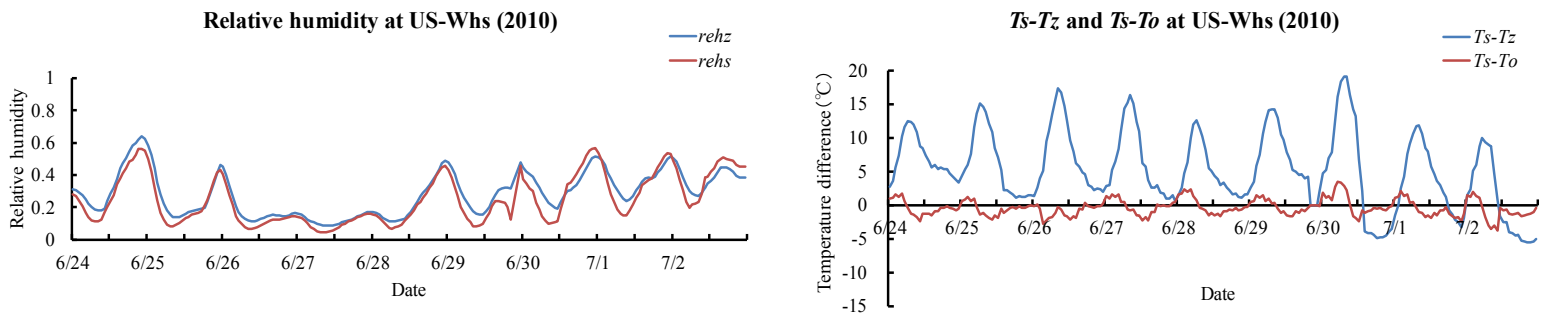

Relative humidity at AU-Dry (2012)

$T s-T z$ and $T s-T o$ at AU-Dry (2012)

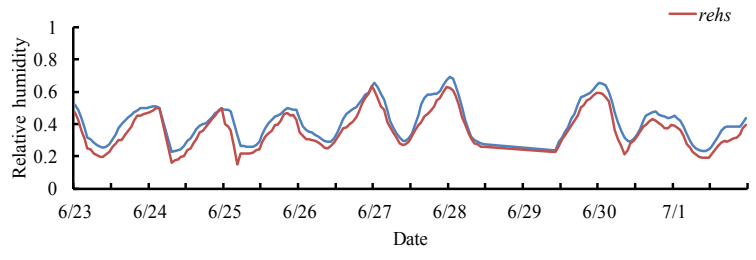

Relative humidity at AU-Stp (2014)
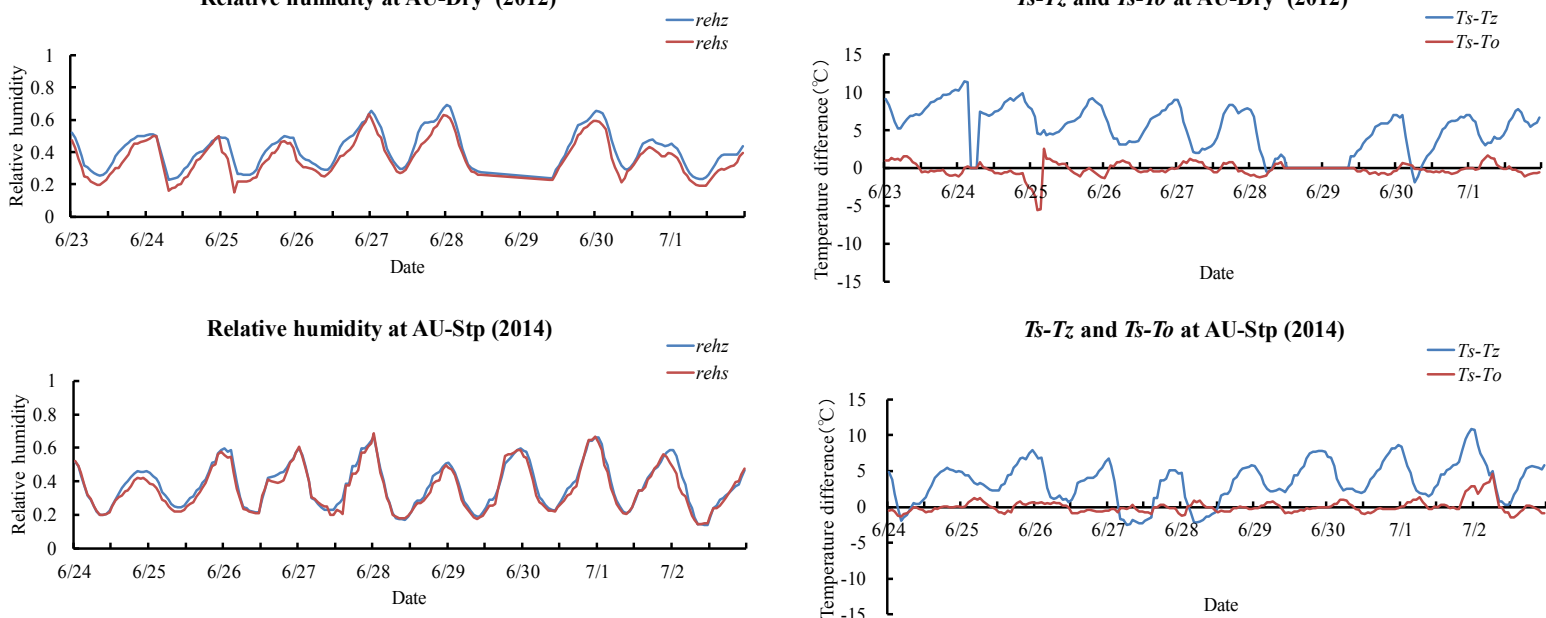

$T s-T z$ and $T s-T o$ at AU-Stp (2014) $-T s-T z$
$-T s-T 0$

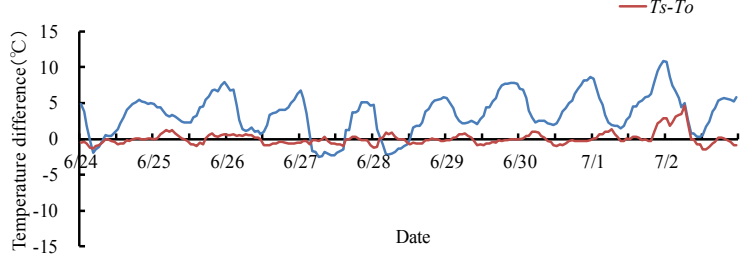

Relative humidity at AU-TTE (2013)

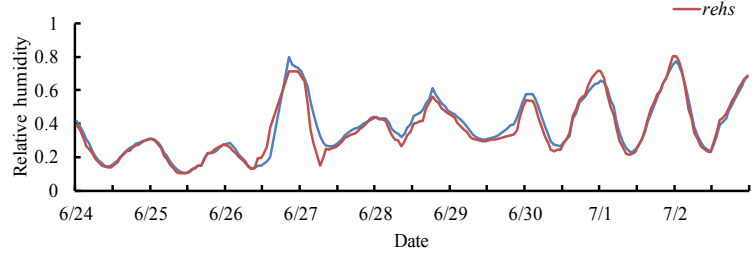

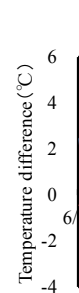

$T s-T z$ and $T s-T o$ at AU-TTE (2013)

$-T s-T z$
$-T s-T o$

Relative humidity at FR-Lam (2008)

-rehz

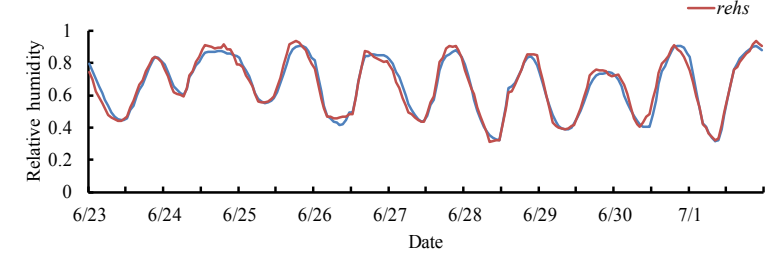



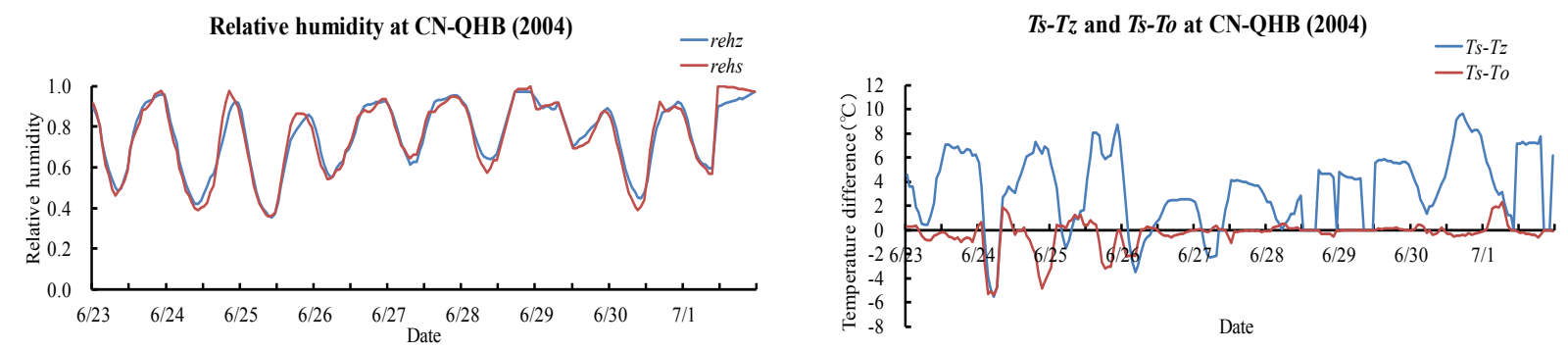

Figure 7. Hourly change of relative humidity and temperature observed and estimated at the tested sites by general method (1) $\left({ }^{\circ} \mathrm{C}\right)$ : The left hand sites describe the comparison of rehs and rehz and right hand side describe the temperature difference $T s-T z$ and $T s-T_{0}$ at all tested sites.

ETa at US-AR2 (2010)

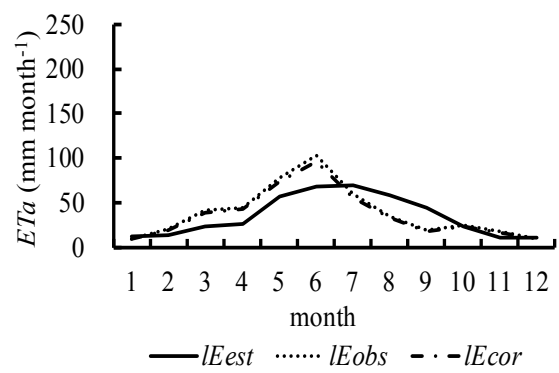

ETa at US-Dia (2012)

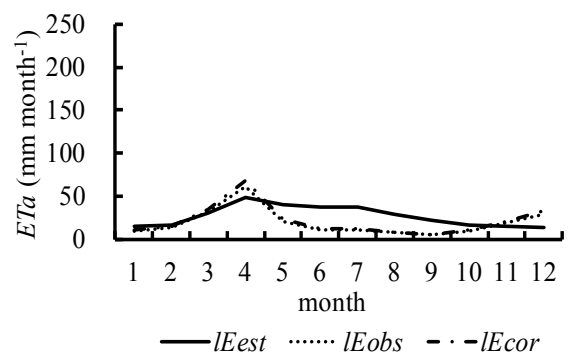

ETa at US-Whs (2010)

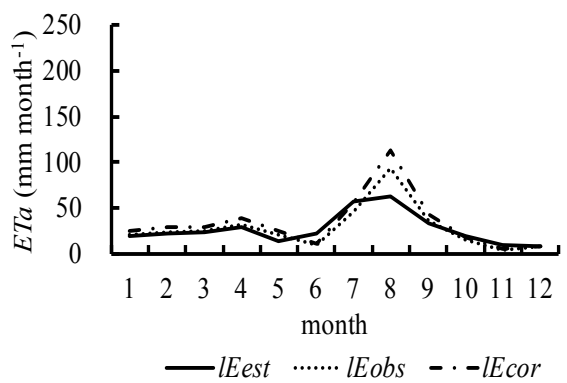

ETa at AU-Dry (2012)

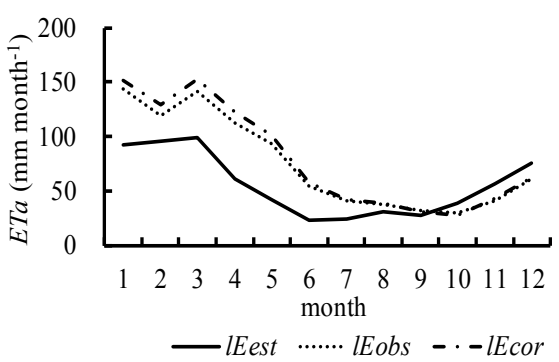

$H a$ at US-AR2(2010)

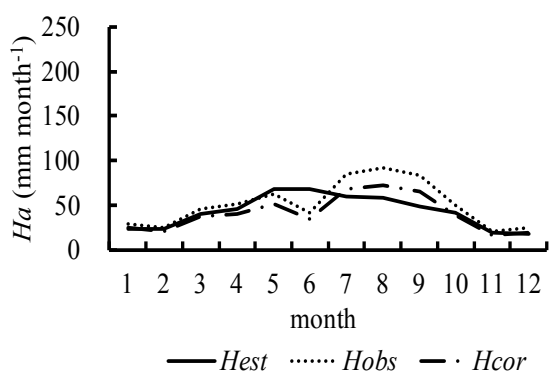

$H a$ at US-Dia (2012)

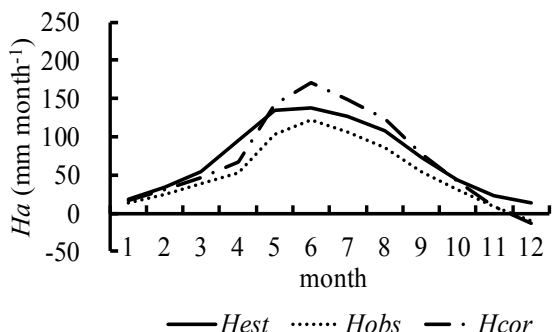

$\mathrm{Ha}$ at US-Whs (2010)

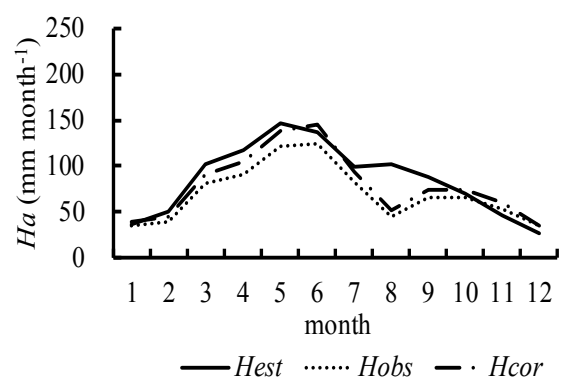

$\mathrm{Ha}$ at AU-Dry (2012)

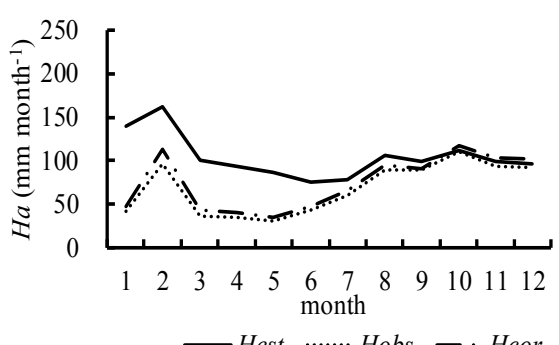


ETa at AU-Stp (2014)

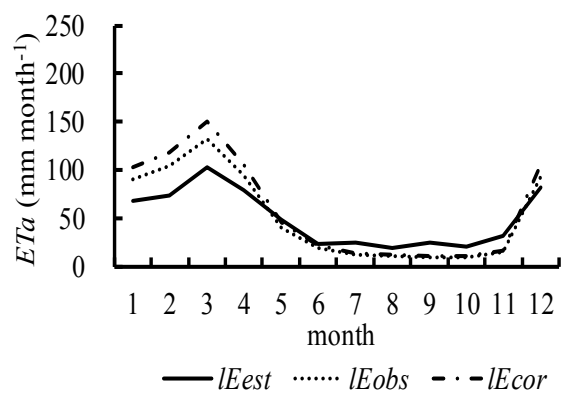

ETa at AU-TTE (2013)

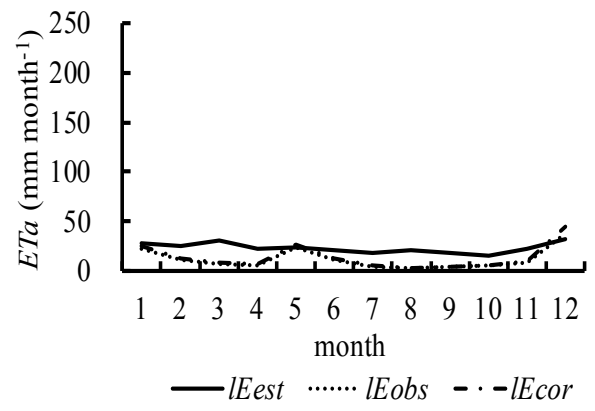

ETa at FR-Lam (2008)

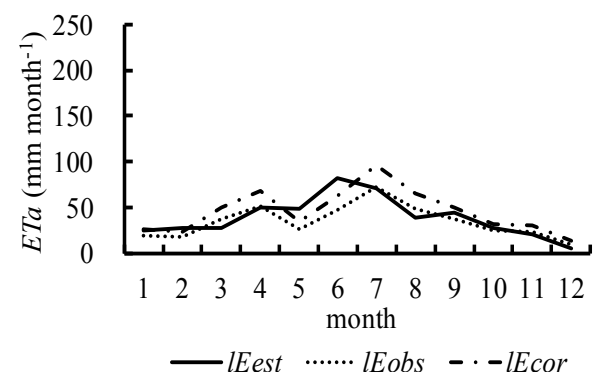

$E T a$ at $\mathrm{CN}-\mathrm{QHB}(2004)$

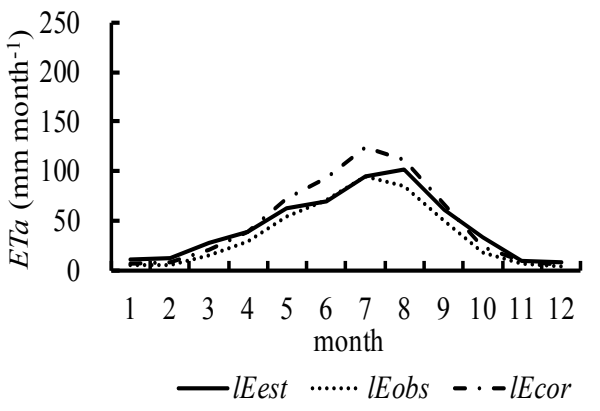

$H a$ at AU-Stp (2014)

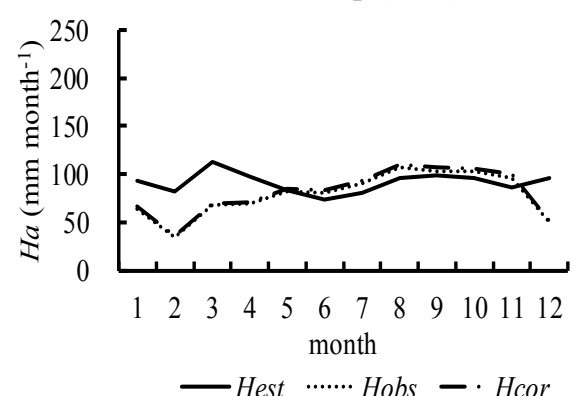

Ha at AU-TTE (2013)

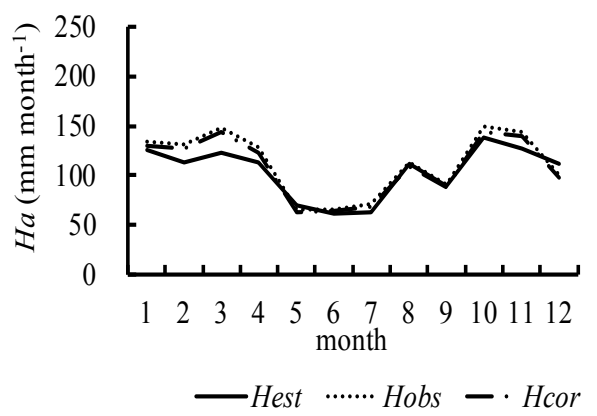

$\mathrm{Ha}$ at FR-Lam (2008)

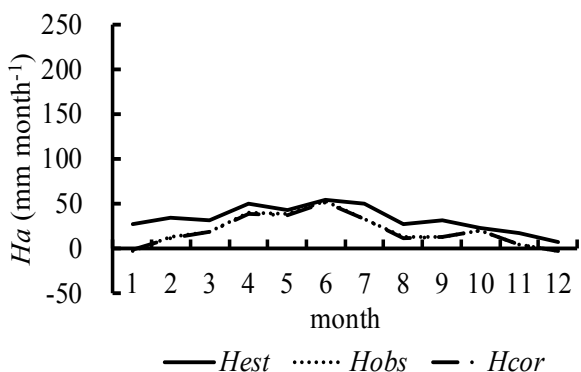

$\mathrm{Ha}$ at $\mathrm{CN}-\mathrm{QHB}$ (2004)

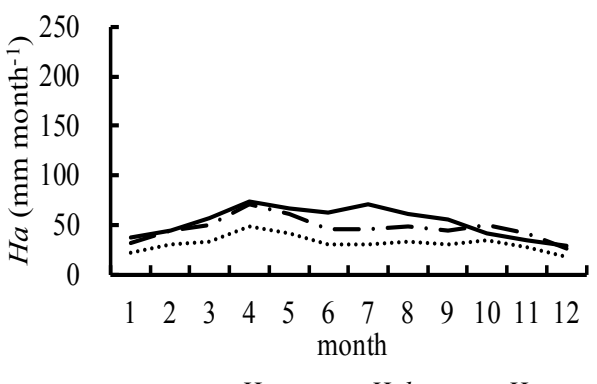

Figure 8. Monthly change of $\mathrm{Ha}$ and $\mathrm{ETa}$ (evapotranspiration) at the tested sites $\left(\mathrm{mm} \cdot \mathrm{month}^{-1}\right)$.

\section{Consideration}

\subsection{Comparison of General Method (1) with Conventional Method}

The conventional method using Equation (3) cannot uniquely determine both $T s$ and rehs because two variables can't determine mathematically by one equation. However, our aim of this analysis is to determine reasonable $I E$ and $H$ values for conserving the heat balance relationship. If we attempt to determine reasonable $I E$ and $H$ values using 
one equation, optimization can be performed by adjusting either $T s$ or rehs to satisfy the relationship. Based on the concept, the following analysis is conducted under initial values of $T s=T z$ and rehs $=$ rehz with the constraint of $0<b<b_{\max }$. Table 5 compares the slope of $I E_{\text {est }}$ and $H_{\text {est }}$ against $I E_{c o r}$ and $H_{c o r}$ (as Figure 6) for general method (1) as well as conventional method.

For $H_{c o r}$, the general method (1) is almost the same accuracy with the conventional method in that the slopes of all sites are adequate range $(1.0 \pm 0.15)$, while for $I E_{c o r}$ all sites under estimate $(<0.85)$. However, conventional method more balanced from the slope of $l E_{c o r}$ and $L E_{c o r}$. In addition, the figures as Figure 4 to Figure 8 in general method (1) that previously described are quite similar those of the conventional method, therefore, the figures abbreviated because of space limitation. In addition, the accuracy criteria $(1.0 \pm 0.15)$ determine conventionally referring to observed data accuracy (Table 3).

Table 5. Comparison of the slope with corrected against estimated for general method (1), conventional method and general method (2): General method (1) estimates $T s$ and rehs using simultaneously two equations as Equation (9) and Equation (10), which guaranteed the uniqueness of variable determination, while conventional method estimate $T s$ and rehs using Equation (3) only which not guaranteed the uniqueness of variable determination. General method (2) used Ts observed by radiometer and rehs determined by Equation (13), thus, guaranteed the uniqueness of variable determination.

\begin{tabular}{|c|c|c|c|c|c|c|c|c|}
\hline \multirow{2}{*}{ Site Name } & \multirow{2}{*}{ Item } & \multicolumn{2}{|c|}{ General meth. (1) } & \multicolumn{2}{|c|}{ Conventional meth. } & \multicolumn{2}{|c|}{ General meth. (2) } & \multirow{2}{*}{ Remarks } \\
\hline & & $I E_{c o r}$ & $H_{\text {cor }}$ & $I E_{c o r}$ & $H_{\text {cor }}$ & $I E_{c o r}$ & $H_{\text {cor }}$ & \\
\hline \multirow[t]{2}{*}{ US-AR2 } & Slope & 0.779 & ${ }^{\star} 0.895$ & 0.808 & $\star 0.865$ & 0.834 & 0.834 & $b>0$ \\
\hline & $R^{2}$ & 0.434 & 0.357 & 0.434 & 0.407 & 0.438 & 0.146 & \\
\hline \multirow[t]{2}{*}{ US-Dia } & Slope & 0.721 & ${ }^{\star} 0.878$ & 0.817 & 0.799 & & & $b>0$ \\
\hline & $R^{2}$ & -0.637 & 0.815 & -0.726 & 0.780 & & & \\
\hline \multirow[t]{2}{*}{ US-Whs } & Slope & 0.655 & ${ }^{*} 1.036$ & 0.803 & ${ }^{*} 0.940$ & & & $b>0$ \\
\hline & $R^{2}$ & 0.598 & 0.745 & 0.519 & 0.743 & & & \\
\hline \multirow[t]{2}{*}{ AU-Dry } & Slope & 0.639 & 1.167 & 0.793 & ${ }^{\star} 0.957$ & ${ }^{\star} 0.864$ & 0.847 & $b>0$ \\
\hline & $R^{2}$ & 0.644 & 0.492 & 0.553 & 0.671 & 0.506 & 0.597 & \\
\hline \multirow[t]{2}{*}{ AU-Stp } & Slope & 0.699 & ${ }^{\star} 1.034$ & 0.717 & ${ }^{\star} 0.983$ & 0.723 & ${ }^{*} 1.012$ & $b>0$ \\
\hline & $R^{2}$ & 0.654 & 0.083 & 0.545 & 0.063 & 0.559 & 0.051 & \\
\hline \multirow[t]{2}{*}{ AU-TTE } & Slope & 0.721 & ${ }^{\star} 0.904$ & ${ }^{\star} 0.963$ & 0.815 & ${ }^{\star} 1.122$ & 0.767 & $b>0$ \\
\hline & $R^{2}$ & -0.951 & 0.822 & -1.574 & 0.814 & -0.907 & 0.694 & \\
\hline \multirow[t]{2}{*}{ FR-Lam } & Slope & 0.764 & ${ }^{\star} 0.984$ & 0.815 & ${ }^{\star} 0.881$ & 0.835 & 0.848 & Without $b$ \\
\hline & $R^{2}$ & 0.502 & 0.167 & 0.500 & 0.148 & 0.510 & 0.118 & \\
\hline \multirow[t]{2}{*}{$\mathrm{CN}-\mathrm{QHB}$} & Slope & 0.843 & ${ }^{*} 1.086$ & ${ }^{\star} 0.856$ & ${ }^{\star} 1.067$ & ${ }^{\star} 0.907$ & ${ }^{\star} 0.979$ & Without $b$ \\
\hline & $R^{2}$ & 0.863 & 0.589 & 0.887 & 0.620 & 0.876 & 0.575 & \\
\hline \multirow[t]{2}{*}{ Average } & Slope & 0.728 & 0.998 & 0.822 & 0.913 & 0.881 & 0.881 & \\
\hline & $R^{2}$ & 0.263 & 0.509 & 0.142 & 0.531 & 0.330 & 0.364 & \\
\hline
\end{tabular}

Note: ${ }^{*}$ indicate accuracy; $(1.0 \pm 0.15)$. 


\subsection{Comparison of General Method (1) with General Method (2) Using Ts Observed by Radiometer}

To verify the validity of our method, estimation $I E$ and $H$ using observed $T s$ ( $T S_{r a d}$ ) by radiometer is performed. By using the $T s$, the $I E$ and $H$ can be determined uniquely by Equation (1) to Equation (3) using $T_{\text {srad }}$ instead of $T_{\text {sass }}$ because the unknown variable is only one. The result described in Table 5 in that this method almost the same accuracy those of conventional method and more balanced $H_{c o r}$ and $l E_{c o r}$. In addition, the result as Figures 4-8 in general method (1) previously described are quite similar those of this method. Therefore, the figures abbreviated because of space limitation.

\subsection{Comparison of Total Amount of Yearly $\mathrm{Ha}$ and ETa of Three Methods}

To compare the total amount of sensible heat ( $\mathrm{Ha}$ ) and latent heat flux (ETa) estimated and corrected expressed in mm.year ${ }^{-1}$ base. The estimation of $\mathrm{Ha}$ and ETa performed three ways as general method (1), conventional method and general method (2). The results summarized in Table 6 .

Table 6 describes that the estimated $H a$ and ETa by the three methods mostly reproduced the corrected Ha and ETa. But there are site specific and method specific difference. Among this, general method (2) is seemed to be mostly suitable because which more coincided with the $H a_{c o r}$ and $E T a_{c o r}$. However, the data of $T_{\text {srad }}$ is rarely at common climate observation, thus, we focused on general method (1) and conventional method.

Table 6. Comparison of corrected Ha and Eta versus estimated Ha and Eta of various method (mm.year $\left.{ }^{-1}\right)$ : General meth. (1), conventional meth. and general meth. (2) refer to the caption of Table 5. Unit of heat flux $\left(\mathrm{W} \cdot \mathrm{m}^{-2}\right)$ converted to $\left(\mathrm{mm} \cdot \mathrm{day}^{-1}\right)$ as ratio of $100\left(\mathrm{~W} \cdot \mathrm{m}^{-2}\right)$ is equivalent for $3.53\left(\mathrm{~mm} \cdot\right.$ day $\left.^{-1}\right)$ [15]. FR-Lam and CN-QHB does not apply $b>0$.

\begin{tabular}{|c|c|c|c|c|c|c|c|c|c|}
\hline \multirow{2}{*}{ Site name } & \multicolumn{2}{|c|}{ Corrected data } & \multicolumn{2}{|c|}{ General method (1) } & \multicolumn{2}{|c|}{ Conventional method } & \multicolumn{2}{|c|}{ General method (2) } & \multirow{2}{*}{ Remarks } \\
\hline & $H a_{c o r}$ & $E T a_{c o r}$ & $H a_{\text {est }}$ & $E T a_{e s t}$ & $H a_{\text {est }}$ & $E T a_{e s t}$ & $H a_{\text {est }}$ & $E T a_{e s t}$ & \\
\hline \multirow[t]{2}{*}{ US-AR2 } & 492 & 434 & 514 & 409 & 517 & 433 & 524 & 427 & $b>0$ \\
\hline & & & ${ }^{\star} 1.04$ & ${ }^{\star} 0.94$ & ${ }^{\star} 1.05$ & ${ }^{\star} 1.00$ & ${ }^{\star} 1.06$ & ${ }^{\star} 0.98$ & \\
\hline \multirow[t]{2}{*}{ US-Dia } & 861 & 256 & 862 & 321 & 759 & 389 & & & $b>0$ \\
\hline & & & ${ }^{\star} 1.00$ & 1.25 & ${ }^{\star} 0.88$ & 1.52 & - & - & \\
\hline \multirow[t]{2}{*}{ US-Whs } & 953 & 403 & 1020 & 322 & 892 & 410 & & & $b>0$ \\
\hline & & & ${ }^{\star} 1.07$ & 0.80 & ${ }^{\star} 0.94$ & ${ }^{\star} 1.02$ & - & - & \\
\hline \multirow[t]{2}{*}{ AU-Dry } & 901 & 957 & 1247 & 666 & 1184 & 871 & 1079 & 978 & $b>0$ \\
\hline & & & 1.38 & 0.70 & 1.31 & ${ }^{\star} 0.91$ & 1.20 & ${ }^{\star} 1.02$ & \\
\hline \multirow[t]{2}{*}{ AU-Stp } & 978 & 710 & 1098 & 596 & 1097 & 679 & 1125 & 656 & $b>0$ \\
\hline & & & ${ }^{\star} 1.12$ & 0.84 & *1.12 & ${ }^{\star} 0.96$ & ${ }^{\star} 1.15$ & ${ }^{\star} 0.92$ & \\
\hline \multirow[t]{2}{*}{ AU-TTE } & 1297 & 165 & 1245 & 277 & 1144 & 403 & 1093 & 455 & $b>0$ \\
\hline & & & ${ }^{\star} 0.96$ & 1.68 & ${ }^{\star} 0.88$ & 2.44 & 0.84 & 2.76 & \\
\hline \multirow[t]{2}{*}{ FR-Lam } & 236 & 555 & 396 & 469 & 359 & 482 & 350 & 491 & $\begin{array}{c}\text { Without } \\
b\end{array}$ \\
\hline & & & 1.68 & ${ }^{*} 0.85$ & 1.52 & ${ }^{\star} 0.87$ & 1.48 & ${ }^{\star} 0.88$ & \\
\hline \multirow[t]{2}{*}{$\mathrm{CN}-\mathrm{QHB}$} & 620 & 618 & 636 & 531 & 685 & 577 & 631 & 631 & $\begin{array}{c}\text { Without } \\
b\end{array}$ \\
\hline & & & ${ }^{\star} 1.03$ & ${ }^{\star} 0.86$ & ${ }^{\star} 1.1$ & ${ }^{\star} 0.93$ & ${ }^{\star} 1.02$ & ${ }^{\star} 1.02$ & \\
\hline
\end{tabular}

Note: second row of each sites indicate the ratio against observed (corrected) data. Note: ${ }^{*}$ indicate the accuracy; (1.0 \pm 0.15 ). Note: observed data obtained by general solution (1). 
To make clearer the accuracy, the annual $\mathrm{Ha}$ and $E T a$, compared as the ratio of estimated $H_{e s t}$ and $E T a_{e s t}$ against corrected $H a_{c o r}$ and $E T a_{c o r}$. Which shown in second row of each sites in Table 6 because the corrected data is a standard of reasonable estimation.

Attached star indicate the accuracy range (1.0 \pm 0.15$)$. For general method (1), most of $H a_{e s t} / H a_{c o r}$ indicated $(1.0 \pm 0.15)$ except FR-Lam while over half sites of $E T a_{e s t} / E T a_{c o r}$ indicate out of $(1.0 \pm 0.15)$. For conventional method, most of $H a_{e s t} / H a_{c o r r}$, indicate under 0.15 except AU-dry and FR-Lam while $E T a_{e s t} / E T a_{c o r}$ all sites indicate under $0.15 \mathrm{ex}-$ cept US-Dia and AU-TTE where are belong to arid region. For general method (2), $H \mathrm{a}_{\text {est }} / \mathrm{Ha}_{\text {cor }}$ and $E T a_{\text {est }} / E T \mathrm{a}_{\text {cor }}$ at three sites indicate $(1.0 \pm 0.15)$ but AU-TTE and AU-Dry and FR-Lam of $\mathrm{Ha}_{e s t}$ are out of $(1.0 \pm 0.15)$. All of the above data describe mostly reasonable against our proposed method, although there are some exceptions.

\subsection{Comparison of ETa versus Precipitation from the Aspect of Water Balance}

It is considered that annual precipitation almost consumed as ETa in arid and semiarid region. Figure 9 describes the relationship of annual precipitation versus $E T a_{c o r}$ and $E_{T a_{e s t}}$ by general method (1). Both figures describe mostly consistent each other. The facts indicate the reasonability of our proposed method from the aspect of water balance.

\section{$E T a_{c o r}$ Versus Prec.}

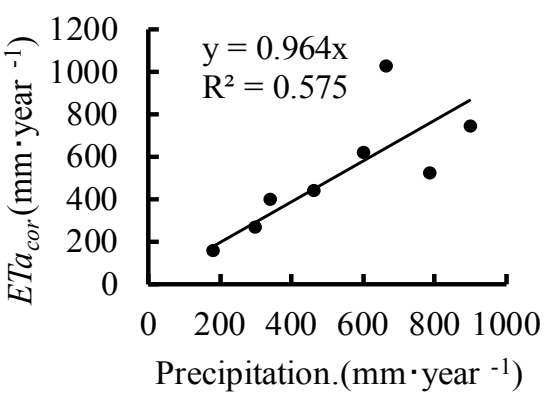

$E T a_{\text {est }}$ Versus Prec.

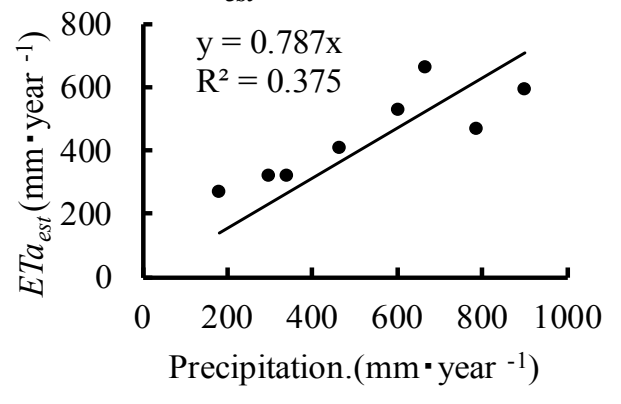

Figure 9. Comparison of ETa $a_{c o r}$ and ETa est versus precipitation.

\section{Discussion}

\subsection{Effect of Constraints " $b$ " on $l E / H$ Ratio}

The constraint expressed by Equation (15) is very well functioned for estimation of $I E$ and $H$ in arid region as US-Dia and AU-TTE having annual precipitation $297 \mathrm{~mm}$ and $180 \mathrm{~mm}$. If the constraint does not apply at AU-TTE, for example, slope of $I E_{\text {est }} / I E_{\text {cor }}$ is 1.552, while $H_{\text {est }} H_{\text {cor }}$ is 0.586 (not shown in figure or table). But if it applied the constraint at the same site, the slope of $I E_{\text {est }} I E_{\text {cor }}$ is 0.721 while $H_{\text {est }} / H_{\text {cor }}$ is 0.904 , resulting in remarkably improved. Apparent meaning of the constraint is almost rehs $<$ rehz, but if the constraint applied directly the $H_{\text {est }}$ and $I E_{\text {est }}$ do not achieve optimal value smoothly.

Moreover, this constraint does not well functioned at semi-arid region having relatively large amount of precipitation as FR-Lam and CN-Hab. Therefore, the two sites do not apply the constraints. The other sites as AU-Dry, AU-Stp, US-Ar2 and US-Whs are intermediate of above sites. If the constraint applied, the $H_{\text {est }}$ are well estimated 
while if do not applied the $I E_{\text {est }}$ are well estimated.

\subsection{Exceptions of the Abnormal Fluctuation in Convergence Process}

In convergence process, The $H_{\text {est }}$ and $I E_{\text {est }}$ sometimes fluctuate plus or minus abnormally because sum of those is limited $(=R n+G)$. For example, the former estimate abnormally large (plus) while the latter abnormally small (minus). To avoid the abnormal fluctuation, constraint as $\mathrm{ABS}(R n+G)<H$ or $I E$ is applied. On the other hand, observed data contain sometime the abnormal data that appear at near $0^{\circ} \mathrm{C}$ in winter season or early morning. The analyses were conducted without the abnormal data [1] [2].

\section{Conclusions}

In the natural world, air temperature and humidity reflect the partitioning of the sensible and latent heat flux from $R n$ and $G$. Based on the concepts, we attempt to reciprocally estimate the $H$ and $I E$ from $R n$ and $G$. By applying the Bowen ratio concept to the soil surface, the unknown variables $T s$ and $q(T s)$, i.e., rehs, are estimated by an optimization procedure satisfying the heat balance relationship [1] [2].

This method is very effective to expand the utility of the water recourses issues through estimation of evapotranspiration for various areas because it requires only single height $T z$ and rehz that are very popular climate elements.

The validation of the method was conducted using eight globally dispersed sites, where observed $I E$ and $H$ by the eddy covariance using FLUXNET. The analysis was conducted on an hourly basis and was summarized as daily averages.

The analysis was conducted using three methods, namely, the general (1), conventional and general (2) methods. The general method (1) is based on the unified determination of $T s$ and rehs by solving two heat balance equations simultaneously, whereas the conventional method is based on solving a single heat balance equation that does not unify $T s$ and rehs. The general method (2) is based on $T s$ observed by radiometer.

The general method (1) is mathematically reasonable, but the data of two unit times are required. The conventional method does not allow for the uniqueness of $T s$ and rehs, but reasonable partitioning of $H$ and $I E$ can be achieved by adjusting either $T s$ or rehs, depending on the GRG algorithm. The main purpose of our research is to accurately estimate $I E$ and $H$ rather than to accurately estimate $T s$ and rehs; thus, the conventional method can be used as well as the general method (1). If $T s$ observed by radiometer, application of the general method (2) is reasonable.

The main validated results are as follows:

1) The observed data are corrected by regression analysis because it does not guarantee the heat balance relationship.

2) The general and conventional solutions are nearly consistent for hourly changes of $I E_{\text {est }}$ with $I E_{\text {cor }}$ as well as $H_{\text {est }}$ with $H_{\text {cor }}$ at eight sites. However, there are some sitespecific differences.

3) The general and conventional solutions are nearly consistent for the annual changes of $I E_{\text {est }}$ with $I E_{c o r}$ and $H_{\text {est }}$ with $H_{c o r}$ at eight sites. However, there are some seasonal and site-specific differences.

4) Analysis of annual change of Ha and ETa are performed. The results describe that 
$H a_{e s t}$ is consistent with $H a_{\text {cor }}$ and $E T a_{\text {est }}$ consistent with $E T a_{\text {cor }}$.

5) To confirm the reasonability of general method (1), conventional method and general method (2), the ratio of $E T a_{\text {est }} / E T a_{\text {cor }}$ is compared. The result indicates that there is no remarkable difference among the two methods.

6) Annual precipitation is mostly consistent with $E T a_{\text {est }}$ and $E T a_{c o r}$ from the aspect of water balance.

The estimated results do not completely reproduce the observed data, but the results are mostly satisfactory for the estimation of $I E$ and $H$. The remarkable feature of this method is that it is applicable for single height of temperature and humidity with $R n$ and $G$. This feature shows the method would be widely applicable for estimation of $I E$. If this procedure is approved widely, the resolution of water resources problem and reasonable irrigation planning will be more advanced.

However, there are some issues to be solved in future: 1) Error plain of $\varepsilon$ in Equation (3) or Equation (9) and Equation (10), i.e., relationship of $\mathcal{E}$ with $T s$ and rehs, is very complicated, having many local minimum. Therefore, the importance of determination of initial values is still important; 2) Applying constraint is also important issues because it strongly affects the results; 3) Snow and frozen problems are not considered in this research so that these problems should be solved in future and 4) Accuracy of the observed data is also very important issue which has been improved nowadays by many researchers and technologists.

We conclude that $E T$ and $H$ are controlled by energy conservation in nature. Realistically, the observed temperature and humidity are strongly affected by the partitioning of $H$ and $I E$ and vice versa. Therefore, using the observed temperature, humidity and common climate elements, $I E$ and $H$ values are reciprocally approximated by the optimized techniques [1] [2].

\section{Acknowledgements}

We express sincere thanks to the AmeriFlux, EuroFlux and AsiaFlux principal investigation for data accessed July 5, 2015. We thank Dr. Fujihara Yooich and Dr. Takimoto Hiroshi for providing valuable comments for the optimization procedure. We acknowledge the following AmeriFlux sites for their data records: site IDs. In addition, funding for AmeriFlux data resources was provided by the U.S. Department of Energy's Office of Science.

\section{References}

[1] Maruyama, T. and Segawa, M. (2016) Reciprocal Analysis of Sensible and Latent Heat Fluxes in a Forest Region Using Single Height Temperature and Humidity Based on the Bowen Ratio Concept. Journal of Water Resource and Protection, 8, 724-742. https://doi.org/10.4236/jwarp.2016.87059

[2] Maruyama, T. and Segawa, M. (2016) Application of the Reciprocal Analysis for Sensible and Latent Heat Fluxes with Evapotranspiration at a Humid Region. Journal of Mordan Hydrology, 6, 230-252. https://doi.org/10.4236/ojmh.2016.64019

[3] Kondo, J. (1996) Water and Heat Balance on Soil Surface. In: Kondo, J., Ed., Meteorology on Water Environment, Asakura Publishing Ltd., Tokyo, 128-159.

[4] Twine, T.E., Kustas, W.P., Norman, J.M., Cook, D.R., Houser, P.R., Meyers, T.P., Prueger, 
J.H., Starks, P.J. and Wesely, M.L. (2000) Correcting Eddy-Covariance Flux Underestimates over a Grassland. Agricultural and Forest Meteorology, 103, 279-300.

https://doi.org/10.1016/S0168-1923(00)00123-4

[5] Wilson, K., et al. (2002) Energy Balance Closure at FLUXNET Sites. Agricultural and Forest Meteorology, 113, 223-243. https://doi.org/10.1016/S0168-1923(02)00109-0

[6] Allen, R. (2008) Quality Assessment of Weather Data and Micrometeorological Flux Impact on Evapotranspiration Calculation. Journal of Agricultural Meteorology, 64, 191-204. https://doi.org/10.2480/agrmet.64.4.5

[7] Beringer, J., Cunningham, S. and Hutley, L. (2014) Sturt Plains Ozflux L2 Data.

[8] Billesbach, D.B., Torn, J.A. and Margaret, S. (2010) ARM USDA UNL OSU Woodward Switchgrass 2. US-Br3 AmeriFlux L2 Data.

http://cdiac.esd.ornl.gov/programs/ameriflux/data system/aaARM USDA UNL OSU Wo odward Switchgrass 2 pf.html

[9] Ceschia, E. and Tallec, T. (2008) Lamasquere (FR-Lam) European Fluxes Database Cluster L2 Data.

[10] Eamus, D. and Cleverly, J. (2013) Ti Tree East Ozflux L2 Data.

[11] Beringer, J. and Hutley, L. (2010) Dry River Ozflux L2 Data.

[12] Scott, R. (2014) Lucky Hills Shrubland. US-Whs AmeriFlux L2 Data. http://cdiac.esd.ornl.gov/programs/ameriflux/data system/aaLucky Hills Shrubland pf.ht $\underline{\mathrm{ml}}$

[13] Wharton, S. (2012) Diablo. US-Dia AmeriFlux L2 Data. http://cdiac.esd.ornl.gov/programs/ameriflux/data system/aaDiablo_pf.html

[14] Tang, Y., Du, M. and Kato, T. (2004) Qinghai Flux Research Site. CN-QHB AsiaFlux L2 Data.

[15] Kondo, J. (2015) Heat Balance and Climate on Soil Surface. 


\section{Appendix 1}

The GRG Nonlinear Solving Method for nonlinear optimization: developed by Leon Lasdon (University of Texas at Austin) and Alan Waren (Cleveland State University) and enhanced by Frontline Systems, Inc.

For more information about the other solution algorithms, advice on building effective solver models, and solving larger scale problems, contact: Frontline Systems, Inc.

Web site: http://www.solver.com, E-mail: info@solver.com

Eestimated results have not completely reproduced the observations, but the results are mostly satisfaction

\section{Appendix 2}

Using modules of Visual Basic for Applications (VBA) in the manuscript Sub Macro "Number1 ( )

'Macro "Number 1": GRG method

Dim $r$ As Long

Dim lastRow As Long

lastRow $=$ Range (“〈Column Alphabet $\rangle$ " \& Rows Count).End (xlUp).Row

SolverReset

For $r=\langle$ Start row number $\rangle$ To 〈End row number $\rangle$

SolverReset

SolverOptions Precision: $=0.000001$, Convergence $=0.0001$, StepThru: $=$ False, Scaling:=False

, AssumeNonNeg:=False, Derivatives: $=2$

SolverOkSetCell:= "Row" \& r, MaxMinVal:=2, ValueOf:=0_

, ByChange:=Range(Cells(r, 〈First column number〉), Cells(r, 〈Last column number〉))

SolverAddCellRef:="\$ 〈rehs's Column Alphabet $\rangle$ " \& r, Relation:=1, FormulaText:=1

SolverAddCellRef:="\$ 〈rehs's Column Alphabet $\rangle$ " \& r, Relation:=3, FormulaText:=0

SolverAddCellRef:="\$ $\langle R T$ s's Column Alphabet $\rangle$ " \& $\mathrm{r}$, Relation:=1, FormulaText:=5

SolverAddCellRef:="\$ $\langle R T s$ 's Column Alphabet $\rangle$ " \& r, Relation:=3, FormulaText:=5

SolverAddCellRef:="\$ 〈Hestimated's Column Alphabet〉 " \& r, Relation:=1, FormulaText:= "\$ $\langle R n-G$ observed's Column Alphabet $\rangle$ \$ \&r

SolverAddCellRef:="\$ 〈Hestimated's Column Alphabet〉 " \& r, Relation:=3, FormulaText: $=-100$

SolverAddCellRef:="\$ 〈LEestimated's Column Alphabet〉 " \& r, Relation:=1, FormulaText:= "\$ $\langle R n-G$ observed's Column Alphabet $\rangle$ \$ \&

SolverAddCellRef:="\$ 〈LEestimated's Column Alphabet $\rangle$ " \& r, Relation:=3, Formu-

laText: $=-100$

SolverAddCellRef:=" $\left\langle B_{a p p}\right.$ 's Column Alphabet $\rangle$ " \& r, Relation:=1, FormulaText:=100

SolverAddCellRef:=" $\left\langle\left\langle B_{a p p}\right.\right.$ 's Column Alphabet $\rangle$ " \& r, Relation:=3, FormulaText:=100

※in case of $0<b<b_{\max }$ 


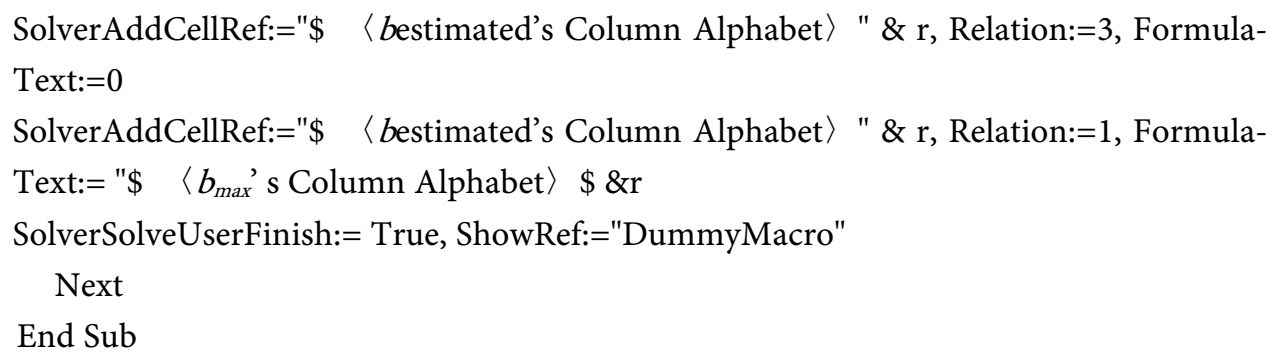

Submit or recommend next manuscript to SCIRP and we will provide best service for you:

Accepting pre-submission inquiries through Email, Facebook, LinkedIn, Twitter, etc. A wide selection of journals (inclusive of 9 subjects, more than 200 journals)

Providing 24-hour high-quality service

User-friendly online submission system

Fair and swift peer-review system

Efficient typesetting and proofreading procedure

Display of the result of downloads and visits, as well as the number of cited articles Maximum dissemination of your research work

Submit your manuscript at: http://papersubmission.scirp.org/

Or contact ojmh@scirp.org 